\title{
Asymptotic Stability of Shock Wave for the Outflow Problem Governed by the One-Dimensional Radiative Euler Equations
}

\author{
Lili Fan ${ }^{1}$, Lizhi Ruan ${ }^{2}$ and Wei Xiang ${ }^{3, *}$ \\ ${ }^{1}$ School of Mathematics and Computer Science, Wuhan Polytechnic \\ University, Wuhan 430023, China. \\ 2 The Hubei Key Laboratory of Mathematical Physics, School of \\ Mathematics and Statistics, Central China Normal University, \\ Wuhan 430079, China. \\ ${ }^{3}$ Department of Mathematics, City University of Hong Kong, \\ Tat Chee Avenue, Kowloon, Hong Kong, China.
}

Received 10 September 2021; Accepted 2 November 2021

\begin{abstract}
This paper is devoted to the study of the asymptotic stability of the shock wave of the outflow problem governed by the one-dimensional radiative Euler equations, which are a fundamental system in the radiative hydrodynamics with many practical applications in astrophysical and nuclear phenomena. The outflow problem means that the flow velocity on the boundary is negative. Comparing with our previous work on the asymptotic stability of the rarefaction wave of the outflow problem for the radiative Euler equations in [6], two points should be pointed out. On one hand, boundary condition on velocity is considered instead of boundary condition on temperature, which induces a perfect boundary condition on anti-derivative perturbations so that boundary estimates on perturbed unknowns are trickily and smoothly established. On the other hand, the rarefaction wave is an expansive wave, while the shock wave is a compressive wave. So we need take good advantages of properties of the shock wave instead. Our investigation on the outflow problem provides a good understanding on the radiative effect and boundary effect in the setting of shock wave.
\end{abstract}

\footnotetext{
${ }^{*}$ Corresponding author. Email addresses: rlz@mail.ccnu.edu.cn (L. Ruan), weixiang@cityu .
} edu.hk (W. Xiang), fll810@live.cn (L. Fan) 
AMS subject classifications: 35B35, 35B40, 35M30, 35Q35, 76N10, 76N15

Key words: Radiative Euler equations, outflow problem, viscous shock wave, asymptotic stability, initial-boundary value problem.

\section{Introduction}

In this paper, we will continue to study the outflow problem governed by the one-dimensional radiative Euler equations, which is the second one of our series of papers on such kind of outflow problem, actually, the first one on the asymptotic stability of the shock wave for the radiative Euler equations with a boundary. The radiative Euler equations are a fundamental system to describe the motion of the compressible gas with the radiative heat transfer phenomena, which has many applications in astrophysics and nuclear explosions. Mathematically, the one-dimensional radiative Euler equations in the Eulerian coordinates can be modelled as a hyperbolic-elliptic coupled system of the following form:

$$
\left\{\begin{array}{l}
\rho_{t}+(\rho u)_{x}=0, \\
(\rho u)_{t}+\left(\rho u^{2}+p\right)_{x}=0, \\
\left\{\rho\left(e+\frac{u^{2}}{2}\right)\right\}_{t}+\left\{\rho u\left(e+\frac{u^{2}}{2}\right)+p u\right\}_{x}+q_{x}=0, \\
-q_{x x}+a q+b\left(\theta^{4}\right)_{x}=0,
\end{array}\right.
$$

where $\rho, u, p, e$ and $\theta$ are respectively the density, velocity, pressure, internal energy and absolute temperature of the gas, and $q$ is the radiative heat flux. Positive constants $a$ and $b$ depend only on the gas itself. Like the classic compressible Euler equations, the first three equations in (1.1) stand for the conservation of the mass, momentum and energy respectively. The fourth equation in (1.1) is related to the radiative heat transfer phenomenon, and one can refer $[1,9,23,28,39,44]$ for more details. System (1.1) can also be derived by the non-relativistic limit (speed of light tending to $+\infty$ ) from a hyperbolic-kinetic system, and rigorous mathematical derivation can be found in [15].

Precisely speaking, we will investigate the initial-boundary value problem of system (1.1) in the half space $\{(x, t) \mid 0 \leq x, t<\infty\}$ with the initial data

$$
(\rho, u, \theta)(x, 0)=\left(\rho_{0}, u_{0}, \theta_{0}\right)(x) \text { for } \quad x \geq 0,
$$

satisfying

$$
\inf _{x \in[0,+\infty)}\left(\rho_{0}, \theta_{0}\right)(x)>0
$$


and the asymptotic boundary condition at the far field $x=+\infty$

$$
(\rho, u, \theta, q)(+\infty, t)=\left(\rho_{+}, u_{+}, \theta_{+}, 0\right), \quad t \geq 0 .
$$

In what follows, we give a roughly classification of the time-asymptotic states of the solution $(\rho, u, \theta, q)(x, t)$ based on the boundary data $(\rho, u, \theta, q)(0, t)$. It is expected that as time tends to the infinity, the solution is asymptotically described by one of the following waves, such as viscous shock wave, stationary wave, rarefaction wave or the superposition of stationary wave and rarefaction wave. As shown in the Fig. 1, we state some cases which have been solved already or will possibly be solved in the future.

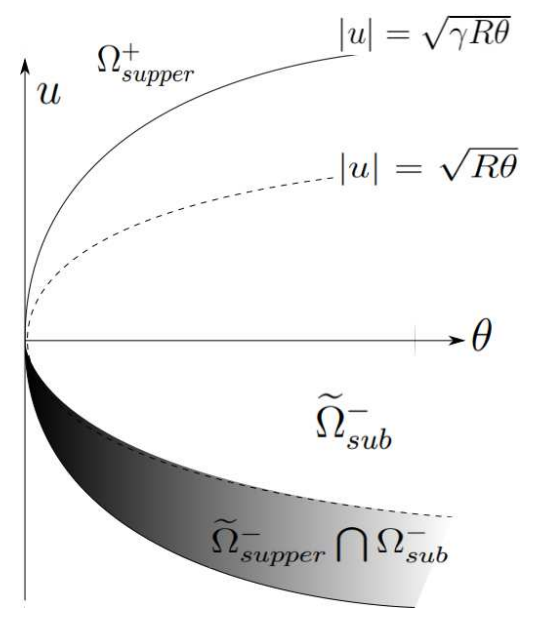

Figure 1:

Case I: inflow problems $(u(0, t)>0)$.

If

$$
\left(\rho_{+}, u_{+}, \theta_{+}\right) \in \Omega_{\text {supper }}^{+}:=\{(\rho, u, \theta) ; u>\sqrt{\gamma R \theta}\},
$$

the boundary condition is given as

$$
(\rho, u, \theta, q)(0, t)=\left(\rho_{-}, u_{-}, \theta_{-}, 0\right) .
$$

Then

(i) If $\left(\rho_{+}, u_{+}, \theta_{+}\right)$locates on the 3 -rarefaction curve past through $\left(\rho_{-}, u_{-}, \theta_{-}\right)$, then there exists a 3-rarefaction wave to the corresponding Riemann problem which connects $\left(\rho_{-}, u_{-}, \theta_{-}\right)$and $\left(\rho_{+}, u_{+}, \theta_{+}\right)$and the 3 -rarefaction wave solution is asymptotic stable. It has been proved in [5]. 
(ii) If $\left(\rho_{+}, u_{+}, \theta_{+}\right)$locates on the contact discontinuity curve past through $\left(\rho_{-}, u_{-}, \theta_{-}\right)$, then the asymptotic stability of the contact discontinuity wave, under the restriction of the smallness of the wave strength, can be obtained. It has been proved in [7].

Case II: outflow problems $(u(0, t)<0)$.

(i) If

$$
\left(\rho_{+}, u_{+}, \theta_{+}\right) \in \widetilde{\Omega}_{\text {supper }}^{-} \bigcap \Omega_{\text {sub }}^{-}:=\{(\rho, u, \theta) ;-\sqrt{\gamma R \theta}<u<-\sqrt{R \theta}\},
$$

the boundary condition can be given as

$$
(\theta, q)(0, t)=\left(\theta_{-}, 0\right) \text {. }
$$

Under this boundary condition, we can find proper numbers $\left(\rho_{-}, u_{-}\right)=$ $(\rho, u)(0, t)$ to show the asymptotic stability of the rarefaction wave without restrictions on the smallness of the wave strength, which has been proved in [6].

(ii) If

$$
\left(\rho_{+}, u_{+}, \theta_{+}\right) \in \widetilde{\Omega}_{\text {sub }}^{-}:=\{(\rho, u, \theta) ;-\sqrt{R \theta}<u<0\},
$$

the boundary condition can be given as

$$
u(0, t)=u_{-}, \quad q(0, t)=0 .
$$

Moreover, the initial condition (1.2) and the boundary condition (1.7) satisfy the compatibility condition that $u_{0}(0)=u_{-}$at the origin $(0,0)$. Under this boundary condition, we can find proper numbers $\left(\rho_{-}, \theta_{-}\right)=(\rho, \theta)(0, t)$ to show the asymptotic stability of the 3 -shock wave under some small assumptions, which is the theme of this manuscript.

We need to emphasize that the classification of asymptotic states of the radiative Euler equations is very complicated. For the other cases, especially on the transonic region $\widetilde{\Gamma}_{s u b}^{-}:=\{(\rho, u, \theta) ;|u|=\sqrt{R \theta}\}$, the situation is more complicated. They will be investigated in the future.

In this paper, we are interested in studying the asymptotic stability of the viscous shock wave of the outflow problem (1.1)-(1.4) and (1.7). Our main results will be stated in Section 2. Comparing with our previous work on the asymptotic stability of the rarefaction wave to the outflow problem for the radiative Euler equations in [6], two points should be pointed out. On one hand, the rarefaction wave is an expansive wave, while the shock wave is a compressive wave. They are different such that we have to develop a different approach to take good 
advantage of properties of the shock wave instead. On the other hand, we impose a different boundary condition, that concerns velocity instead of temperature, which is a perfect boundary condition on the anti-derivative perturbations so that boundary estimates on perturbed unknowns are trickily and smoothly established. In particular, the boundary condition on temperature $\theta(0, t)=\theta_{-}$is frequently considered for the outflow problem governed by the Navier-Stokes equations such as $[14,26]$. However, different from that boundary condition concerns on temperature, boundary condition (1.7) is reasonable since system (1.1) is of Euler-type. Finally, the determination of the shift is based on tedious and tricky calculations.

Now let us review some related work. As far as we know, so far most of the existing results concern the analysis of the global-in-time existence and stability of the elementary waves for the one-dimensional case.

For the Cauchy problem, the global-in-time existence of solutions around a constant state was shown in [16]. For the analysis of the rarefaction wave, if the initial data is a small perturbation of a given rarefaction wave with small strength, it was proved in [20] that the solutions converge to the rarefaction wave as $t \rightarrow+\infty$. Then the authors in [11] showed that when the absorption coefficient $\alpha$ tends to $+\infty$, the solutions converge to the rarefaction wave with the convergence rate $\alpha^{-\frac{1}{3}}|\ln \alpha|^{2}$, where the absorption coefficient $\alpha$ is defined by the relationship $a=3 \alpha^{2}$ and $b=4 \alpha \sigma$ for positive constants $a, b$ and the Stefan-Boltzmann constant $\sigma$. The asymptotic stability of a single viscous contact wave was proved in [41,42]. The existence and stability for zero mass perturbation of the small amplitude shock profile were respectively studied in [21,22]. The authors in [29] showed the nonlinear orbital asymptotic stability of small amplitude shock profiles for general hyperbolic-elliptic coupled systems of the type modeling the radiative gas. Analysis of large amplitude shock profiles was given in [2,24]. Finally, for the case of composite waves, the stability of the composite wave of rarefaction waves and a viscous contact wave was investigated in [33,43]. Recently the authors in [4] studied the unique global-in-time existence and the asymptotic stability of the composite wave of two viscous shock waves by employing the anti-derivative method.

For the initial-boundary value problem, the study of the inflow and outflow problem is initiated by us recently and systematically to deal with the weak dissipation of the radiative Euler system and the difficulties from boundary effect. Our series of results on the inflow and outflow problem in [5-7] provide a good understanding to radiative effect and boundary effect in the setting of elementary waves such as rarefaction wave and contact discontinuity wave. It is natural that shock wave with boundary effect is considered in present paper, which will 
provide a good understanding to radiative effect and boundary effect in the setting of shock wave. For the introduction of the inflow and outflow problems, one can refer to the paper by Matsumura [25] for more details. There are also many results on the study of the inflow or outflow problem governed by other systems such as the Navier-Stokes system (see [12,13,18,26,30-32,38,40]).

We need to mention that we are also motivated by the related investigations on the simplified model (Hamer model), which gives a good approximation to the radiative Euler equations in a certain physical situation, c.f. [10,19]. The investigations on the simplified model provide a good understanding on the radiative effect. The exhaustive literature list is beyond the scope of the paper, and thus only a few closely related results on wave patterns are mentioned, c.f. [3, 8, 17,34-37]. Interested readers can refer to them and references therein.

The rest of the paper is organized as follows. In Section 2, the viscous shock wave is constructed based on the Riemann problem of the full Euler equations. Properties of viscous shock wave which will be frequently used in this paper and the main theorem of this paper are given. Then series of a priori estimates are established in Sections 3-5 so that our main theorem are proved by combing the local wellposedness theorem.

\section{Mathematical formulation and main results}

In this section, we construct the viscous shock wave for (1.1) and then state our main results.

\subsection{Viscous shock wave}

It is well-known that for any given $\left(\rho_{+}, u_{+}, \theta_{+}\right) \in \widetilde{\Omega}_{s u b}$, there is a curved surface in a small neighbourhood of $\left(\rho_{+}, u_{+}, \theta_{+}\right)$for the solution $\left(\rho_{-}, u_{-}, \theta_{-}\right)$of a shock structure with the shock speed $s_{3}>0$, which satisfies the following RankineHugoniot conditions:

$$
\left\{\begin{array}{l}
-s_{3}\left(\rho_{-}-\rho_{+}\right)+\left(\rho_{-} u_{-}-\rho_{+} u_{+}\right)=0 \\
-s_{3}\left(\rho_{-} u_{-}-\rho_{+} u_{+}\right)+\left(\rho_{-} u_{-}^{2}+p_{-}-\rho_{+} u_{+}^{2}-p_{+}\right)=0 \\
-s_{3}\left[\rho_{-}\left(e_{-}+\frac{1}{2} u_{-}^{2}\right)-\rho_{+}\left(e_{+}+\frac{1}{2} u_{+}^{2}\right)\right] \\
\quad+\left\{\rho_{-} u_{-}\left[e_{-}+\frac{\left(u_{-}\right)^{2}}{2}\right]+p_{-} u_{-}-\rho_{+} u_{+}\left[e_{+}+\frac{\left(u_{+}\right)^{2}}{2}\right]-p_{+} u_{+}\right\}=0
\end{array}\right.
$$


and the entropy condition

$$
u_{+}+\sqrt{R \gamma \theta_{+}}<s_{3}<u_{-}+\sqrt{R \gamma \theta_{-}} .
$$

Let

$$
\Omega\left(z_{+}\right):=\left\{(\rho, u, \theta)||\left(\rho-\rho_{+}, u-u_{+}, \theta-\theta_{+}\right) \mid \leq \bar{\delta}\right\},
$$

where $\bar{\delta}$ is a positive constant depending only on $\left(\rho_{+}, u_{+}, \theta_{+}\right)$. The corresponding viscous 3 -shock wave of (1.1) is expressed by $z^{s}:=\left(\rho^{s}, u^{s}, \theta^{s}, q^{s}\right)\left(x-s_{3} t\right)$, connecting the far field states $\left(\rho^{s}, u^{s}, \theta^{s}, q^{s}\right)( \pm \infty)=\left(\rho_{ \pm}, u_{ \pm}, \theta_{ \pm}, 0\right)$. Substituting $z^{s}(\xi)=$ $\left(\rho^{s}, u^{s}, \theta^{s}, q^{s}\right)(\xi)\left(\xi=x-s_{3} t\right)$ into $(1.1)$, one has

$$
\left\{\begin{array}{l}
-s_{3}\left(\rho^{s}\right)^{\prime}+\left(\rho^{s} u^{s}\right)^{\prime}=0 \\
-s_{3}\left(\rho^{s} u^{s}\right)^{\prime}+\left(\rho^{s}\left(u^{s}\right)^{2}+p^{s}\right)^{\prime}=0 \\
-s_{3}\left\{\rho^{s}\left(e^{s}+\frac{\left(u^{s}\right)^{2}}{2}\right)\right\}^{\prime}+\left\{\rho^{s} u^{s}\left(e^{s}+\frac{\left(u^{s}\right)^{2}}{2}\right)+p^{s} u^{s}\right\}^{\prime}+\left(q^{s}\right)^{\prime}=0 \\
a q^{s}+b\left(\theta^{s}\right)^{\prime}=0, \\
u^{s}(-\infty)=u_{-}, \quad\left(\rho^{s}, u^{s}, \theta^{s}\right)(+\infty)=\left(\rho_{+}, u_{+}, \theta_{+}\right)
\end{array}\right.
$$

where ${ }^{\prime}=\frac{d}{d \xi^{\prime}}, p^{s}=p\left(\rho^{s}, \theta^{s}\right), e^{s}=e\left(\theta^{s}\right), p_{ \pm}=p\left(\rho_{ \pm}, \theta_{ \pm}\right)$and $e_{ \pm}=e\left(\theta_{ \pm}\right)$. Integrating (2.4) with respect to $\xi$ over $[\xi,+\infty)$, we obtain

$$
\left\{\begin{array}{l}
-s_{3}\left(\rho^{s}-\rho_{+}\right)+\rho^{s} u^{s}-\rho_{+} u_{+}=0 \\
-s_{3}\left(\rho^{s} u^{s}-\rho_{+} u_{+}\right)+\rho^{s}\left(u^{s}\right)^{2}+p^{s}-\rho_{+} u_{+}^{2}-p_{+}=0 \\
-s_{3}\left\{\rho^{s}\left[e^{s}+\frac{\left(u^{s}\right)^{2}}{2}\right]-\rho_{+}\left(e_{+}+\frac{u_{+}^{2}}{2}\right)\right\} \\
+\rho^{s} u^{s}\left[e^{s}+\frac{\left(u^{s}\right)^{2}}{2}\right]-\rho_{+} u_{+}\left(e_{+}+\frac{u_{+}^{2}}{2}\right)+p^{s} u^{s}-p_{+} u_{+}=\frac{b}{a}\left[\left(\theta^{s}\right)^{4}\right]^{\prime}
\end{array}\right.
$$

which implies

$$
\begin{aligned}
& \left\{\begin{array}{l}
\rho^{s}-\rho_{+}=-\frac{\rho_{+}}{u^{s}-s_{3}}\left(u^{s}-u_{+}\right), \\
R\left(\theta^{s}-\theta_{+}\right)=-\left(u^{s}-s_{3}-\frac{R \theta_{+}}{u_{+}-s_{3}}\right)\left(u^{s}-u_{+}\right),
\end{array}\right. \\
& \left\{\begin{array}{l}
\left(\theta^{s}\right)^{\prime}=H_{1}\left(\theta^{s}\right), \\
\theta^{s}(-\infty)=\theta_{-}, \quad \theta^{s}(+\infty)=\theta_{+},
\end{array}\right.
\end{aligned}
$$


where

$$
\begin{aligned}
H_{1}\left(\theta^{s}\right):= & \frac{a}{4 b\left(\theta^{s}\right)^{3}}\left[C_{v} \rho_{+}-\frac{R p_{+}}{\left(u^{s}-s_{3}\right)\left(u_{+}-s_{3}\right)-R \theta_{+}}\right]\left(u_{+}-s_{3}\right)\left(\theta^{s}-\theta_{+}\right) \\
& -\frac{a}{4 b\left(\theta^{s}\right)^{3}} \frac{R^{2} \rho_{+}\left(u_{+}-s_{3}\right)^{3}}{2\left[\left(u^{s}-s_{3}\right)\left(u_{+}-s_{3}\right)-R \theta_{+}\right]^{2}}\left(\theta^{s}-\theta_{+}\right)^{2}
\end{aligned}
$$

and

$$
\left(\rho^{s}\right)^{\prime}=-\frac{\rho^{s}}{u^{s}-s_{3}}\left(u^{s}\right)^{\prime}, \quad\left(u^{s}\right)^{\prime}=-\frac{R\left(u^{s}-s_{3}\right)}{\left(u^{s}-s_{3}\right)^{2}-R \theta^{s}}\left(\theta^{s}\right)^{\prime} .
$$

By a straightforward computation, we get the following information on $H_{1}^{\prime}\left(\theta_{+}\right)$ from the entropy condition (2.2) and subsonic condition $-\sqrt{R \theta_{ \pm}}<u_{ \pm}<0$ :

$$
\begin{aligned}
H_{1}^{\prime}\left(\theta_{+}\right) & =\frac{a}{4 b \theta_{+}^{3}}\left[C_{v} \rho_{+}-\frac{R p_{+}}{\left(u_{+}-s_{3}\right)^{2}-R \theta_{+}}\right]\left(u_{+}-s_{3}\right) \\
& =\frac{a}{4 b \theta_{+}^{3}}\left[\frac{R \rho_{+}}{\gamma-1}-\frac{R^{2} \rho_{+} \theta_{+}}{\left(u_{+}-s_{3}\right)^{2}-R \theta_{+}}\right]\left(u_{+}-s_{3}\right) \\
& <-\frac{a R \rho_{+}}{4 b \theta_{+}^{3}} \sqrt{R \gamma \theta_{+}}\left[\frac{1}{\gamma-1}-\frac{R \theta_{+}}{\left(u_{+}-s_{3}\right)^{2}-R \theta_{+}}\right]<0 .
\end{aligned}
$$

Therefore, by the theory of ordinary differential system, there exists a unique solution to system (2.7). Therefore, we find the unique solution to system (2.4). Now we introduce some properties of the solution to system (2.4) which will be used later.

Lemma 2.1. For any fixed $\left(\rho_{+}, u_{+}, \theta_{+}\right) \in \widetilde{\Omega}_{\text {sub }}^{-}$, suppose $\gamma>1, \theta_{+}<\theta_{-}$and $\left(\rho_{-}, u_{-}, \theta_{-}\right) \in$ $\Omega\left(z_{+}\right) \cap \widetilde{\Omega}_{\text {sub }}^{-}$satisfies Rankine-Hugoniot condition (2.1). Then system (2.4) admits a smooth solution $\left(\rho^{s}, u^{s}, \theta^{s}, q^{s}\right)\left(x-s_{3} t\right)$, which is unique up to the spatial shift and satisfies the following properties:

(1) $\left(\rho^{s}\right)^{\prime}<0,\left(u^{s}\right)^{\prime}<0,\left(\theta^{s}\right)^{\prime}<0$;

(2) There exists a positive constant $c$ such that

$$
\begin{aligned}
& \left|\left(\rho^{s}-\rho_{ \pm}, u^{s}-u_{ \pm}, \theta^{s}-\theta_{ \pm}\right)\left(x-s_{3} t\right)\right| \lesssim \delta \mathrm{e}^{-c \delta\left|x-s_{3} t\right|}, \\
& \left|\left(q^{s},\left(\rho^{s}\right)^{\prime},\left(u^{s}\right)^{\prime},\left(\theta^{s}\right)^{\prime},\left(\rho^{s}\right)^{\prime \prime},\left(u^{s}\right)^{\prime \prime},\left(\theta^{s}\right)^{\prime \prime}\right)\left(x-s_{3} t\right)\right| \lesssim \delta^{2} \mathrm{e}^{-c \delta\left|x-s_{3} t\right|,}
\end{aligned}
$$

where $\delta:=\left|\left(u_{+}-u_{-}, \theta_{+}-\theta_{-}\right)\right|$.

The proof of Lemma 2.1 is omitted since the argument is standard. Actually, one can follow the argument for the proof of [27, Lemma 1, pp. 85]. 


\subsection{Anti-derivative perturbation}

The solution of (1.1) is expected to converge to the 3-viscous shock wave as $t \rightarrow+\infty$. Let us consider the case that the initial data $\left(\rho_{0}, u_{0}, \theta_{0}\right)(x)$ is given in a neighborhood of $\left(\rho^{s}, u^{s}, \theta^{s}\right)(x+\alpha-\beta)$ for a large constant $\beta>0$, where the shift $\alpha$ will be determined later. Actually, we require that the viscous shock wave is far from the boundary when $t=0$. Then we should determine the shift $\alpha$ such that the solution $(\rho, u, \theta)$ is expected to tend to $\left(\rho^{s}, u^{s}, \theta^{s}\right)\left(x-s_{3} t+\alpha-\beta\right)$. To do this, we denote the anti-derivative perturbations around the 3-viscous shock wave by $(\Phi, \tilde{\Psi}, \tilde{W}, \Gamma)$ as

$$
\begin{aligned}
& \Phi(x, t)=\int_{x}^{\infty}\left[\rho^{s}\left(y-s_{3} t+\alpha-\beta\right)-\rho(t, y)\right] d y, \\
& \widetilde{\Psi}(x, t)=\int_{x}^{\infty}\left[\left(\rho^{s} u^{s}\right)\left(y-s_{3} t+\alpha-\beta\right)-(\rho u)(t, y)\right] d y, \\
& \widetilde{W}(x, t)=\int_{x}^{\infty}\left\{\rho^{s}\left[C_{v} \theta^{s}+\frac{\left(u^{s}\right)^{2}}{2}\right]\left(y-s_{3} t+\alpha-\beta\right)-\rho\left(C_{v} \theta+\frac{u^{2}}{2}\right)(t, y)\right\} d y, \\
& \Gamma(x, t)=\int_{x}^{\infty}\left[q^{s}\left(y-s_{3} t+\alpha-\beta\right)-q(t, y)\right] d y
\end{aligned}
$$

and denote the perturbation of $(\rho, u, \theta, q)$ around the 3-viscous shock wave by $(\phi, \psi, \zeta, \omega)$, which satisfies

$$
\begin{aligned}
& \phi:=\rho-\rho^{s}=\Phi_{x}, \quad \psi:=u-u^{s}=\frac{1}{\rho}\left(\widetilde{\Psi}_{x}-u^{s} \Phi_{x}\right), \\
& \zeta:=\theta-\theta^{s}=\frac{1}{C_{v} \rho}\left(\widetilde{W}_{x}-\frac{1}{2} \rho \psi^{2}-\rho u^{s} \psi-E^{s} \phi\right), \\
& \omega:=q-q^{s}=\Gamma_{x}
\end{aligned}
$$

where $E^{S}=: C_{v} \theta^{S}+\frac{1}{2}\left(u^{S}\right)^{2}$. It is easy to see that $(\phi, \psi, \zeta, \omega)$ satisfies

$$
\left\{\begin{array}{l}
\phi_{t}+u \phi_{x}+\rho \psi_{x}=Q_{1}, \\
\psi_{t}+u \psi_{x}+R \zeta_{x}+\frac{R \theta}{\rho} \phi_{x}=Q_{2}, \\
C_{v} \zeta_{t}+C_{v} u \zeta_{x}+R \theta \psi_{x}+\omega_{x}=Q_{3}, \\
-w_{x x}+a w+4 b \theta^{3} \zeta_{x}+4 b \theta_{x}^{s} \zeta\left(\theta^{2}+\theta \theta^{s}+\left(\theta^{s}\right)^{2}\right)=q_{x x}^{s},
\end{array}\right.
$$

where

$$
Q_{1}:=-\left(\rho_{x}^{s} \psi+u_{x}^{s} \phi\right)=O(1)\left|\left(\rho_{x}^{s}, u_{x}^{s}\right)\right||(\phi, \psi)|,
$$




$$
\begin{aligned}
& Q_{2}:=-u_{x}^{s} \psi-\rho_{x}^{s}\left(\frac{R \zeta}{\rho}-\frac{R \theta^{s}}{\rho \rho^{s}} \phi\right)=O(1)\left|\left(\rho_{x}^{s}, u_{x}^{s}, \theta_{x}^{s}\right)\right||(\phi, \psi, \zeta)|, \\
& Q_{3}:=-C_{v} \theta_{x}^{s} \psi-R u_{x}^{s} \zeta=O(1)\left|\theta_{x}^{s} \psi+u_{x}^{s} \zeta\right|
\end{aligned}
$$

with

$$
\begin{aligned}
& (\phi, \psi, \zeta)(x, 0)=\left(\phi_{0}, \psi_{0}, \zeta_{0}\right)(x) \rightarrow(0,0,0) \text { as } x \rightarrow+\infty, \\
& \psi(0, t)=u_{-}-u^{s}(-s t+\alpha-\beta), \\
& \omega(0, t)=-q^{s}(-s t+\alpha-\beta) .
\end{aligned}
$$

Introducing the new good unknowns

$$
\Psi=\tilde{\Psi}-u^{s} \Phi, \quad W=\frac{\gamma-1}{R}\left(\tilde{W}-E^{s} \Phi-u^{s} \Psi\right),
$$

we get

$$
\begin{aligned}
& \rho \psi=\Psi_{x}+u_{x}^{s} \Phi, \\
& C_{v} \rho \zeta=C_{v} W_{x}+C_{v} \theta_{x}^{s} \Phi+u_{x}^{s} \Psi-\frac{1}{2} \rho \psi^{2}, \\
& C_{v} W_{x}=C_{v} \rho \zeta-C_{v} \theta_{x}^{s} \Phi-u_{x}^{s} \Psi+\frac{1}{2} \rho \psi^{2} .
\end{aligned}
$$

By a straightforward computation, we have

$$
\left\{\begin{array}{l}
\Phi_{t}+\left(\rho u-\rho^{s} u^{s}\right)=0, \\
\tilde{\Psi}_{t}+\left(\rho u^{2}-\rho^{s} u^{s 2}\right)+\left(p-p^{s}\right)=0 \\
\tilde{W}_{t}+\left\{\rho u\left(e+\frac{u^{2}}{2}\right)-\rho^{s} u^{s}\left[e^{s}+\frac{\left(u^{s}\right)^{2}}{2}\right]\right\}+\left(p u-p^{s} u^{s}\right)=q^{s}-q
\end{array}\right.
$$

which particularly implies that on the boundary $x=0$

$$
\begin{aligned}
& \Phi_{t}(0, t)+u_{-} \phi(0, t)+\left(\rho^{s} \psi\right)(0, t)=0, \\
& \tilde{\Psi}_{t}(0, t)+u_{-}^{2} \phi(0, t)+\rho^{s}\left(u^{s}+u_{-}\right) \psi(0, t)+\left(p-p^{s}\right)(0, t)=0 .
\end{aligned}
$$

Combining (2.20) and (2.21), we further get

$$
\left(\tilde{\Psi}-u_{-} \Phi\right)_{t}(0, t)+\rho^{s} u^{s} \psi(0, t)+\left(p-p^{s}\right)(0, t)=0 .
$$

It follows from the third equation in (2.19) that

$$
\tilde{W}_{t}(0, t)+\frac{1}{2}\left[\rho u^{3}-\rho^{s}\left(u^{s}\right)^{3}\right](0, t)+\frac{\gamma}{\gamma-1}\left(p u-p^{s} u^{s}\right)(0, t)=q^{s}(0, t) \text {. }
$$


By the straightforward computation, it implies

$$
\begin{aligned}
& \tilde{W}_{t}(0, t)+\frac{1}{2} u_{-}^{3} \phi(0, t)+\left[\frac{1}{2} \rho^{s}\left(u_{-}^{2}+u_{-} u^{s}+\left(u^{s}\right)^{2}\right)+\frac{\gamma p^{s}}{\gamma-1}\right] \psi(0, t) \\
& +\frac{\gamma}{\gamma-1} u_{-}\left(p-p^{s}\right)(0, t)=q^{s}(0, t) .
\end{aligned}
$$

Therefore,

$$
\begin{aligned}
& \left(\tilde{W}-\frac{u_{-}^{2}}{2} \Phi\right)_{t}(0, t)+\left[\frac{1}{2} \rho^{s} u^{s}\left(u_{-}+u^{s}\right)+\frac{\gamma p^{s}}{\gamma-1}\right] \psi(0, t) \\
& +\frac{\gamma u_{-}}{\gamma-1}\left(p-p^{s}\right)(0, t)=q^{s}(0, t) .
\end{aligned}
$$

Thus, we get

$$
\left[\tilde{W}-\frac{\gamma u_{-}}{\gamma-1} \tilde{\Psi}+\left(\frac{\gamma}{\gamma-1}-\frac{1}{2}\right) u_{-}^{2} \Phi\right]_{t}(0, t)=-(J \psi)(0, t)+q^{s}(0, t)
$$

where

$$
J:=\frac{1}{2} \rho^{s}\left(u^{s}\right)^{2}+\frac{\gamma}{\gamma-1} p^{s}+\left(\frac{1}{2}-\frac{\gamma}{\gamma-1}\right) \rho^{s} u^{s} u_{-} .
$$

Integrating (2.26) over $[0, t]$, we immediately obtain

$$
\begin{aligned}
& {\left[\tilde{W}-\frac{\gamma u_{-}}{\gamma-1} \tilde{\Psi}+\left(\frac{\gamma}{\gamma-1}-\frac{1}{2}\right) u_{-}^{2} \Phi\right](0, t) } \\
= & {\left[\tilde{W}-\frac{\gamma u_{-}}{\gamma-1} \tilde{\Psi}+\left(\frac{\gamma}{\gamma-1}-\frac{1}{2}\right) u_{-}^{2} \Phi\right](0,0)-\int_{0}^{t}\left(J \psi-q^{s}\right)(0, \tau) d \tau . }
\end{aligned}
$$

We expect that as $t \rightarrow+\infty$,

$$
\left[\tilde{W}-\frac{\gamma u_{-}}{\gamma-1} \tilde{\Psi}+\left(\frac{\gamma}{\gamma-1}-\frac{1}{2}\right) u_{-}^{2} \Phi\right](0, t) \rightarrow 0
$$

That is, if let

$$
\begin{aligned}
I(\alpha):=\tilde{W} & (0,0)-\frac{\gamma u_{-}}{\gamma-1} \tilde{\Psi}(0,0)+\left(\frac{\gamma}{\gamma-1}-\frac{1}{2}\right) u_{-}^{2} \Phi(0,0) \\
& -\int_{0}^{\infty}\left(J \psi-q^{s}\right)(0, \tau, \alpha, \beta) d \tau
\end{aligned}
$$

then we require that

$$
I(\alpha)=0
$$


If (2.30) holds, then by (2.21)-(2.30), we can get the following boundary condition:

$$
\tilde{W}(0, t)-\frac{\gamma u_{-}}{\gamma-1} \tilde{\Psi}(0, t)+\left(\frac{\gamma}{\gamma-1}-\frac{1}{2}\right) u_{-}^{2} \Phi(0, t)=A(t),
$$

where

$$
A(t):=\int_{t}^{\infty}\left(J \psi-q^{s}\right)(0, \tau, \alpha, \beta) d \tau=O(1) \mathrm{e}^{-c \delta \beta} \mathrm{e}^{-c \delta t} .
$$

Now we will determine $\alpha$ such that (2.30) holds. In fact, we will compute the derivative $I^{\prime}(\alpha)$ of $I(\alpha)$ such that the shift $\alpha$ can be determined. Up to now, we have gotten that

$$
\begin{aligned}
I(\alpha)= & \int_{0}^{\infty}\left\{\rho^{s}\left[C_{v} \theta^{s}+\frac{\left(u^{s}\right)^{2}}{2}\right](y+\alpha-\beta)-\rho_{0}\left(C_{v} \theta_{0}+\frac{u_{0}^{2}}{2}\right)(y)\right\} d y \\
& -\frac{\gamma u_{-}}{\gamma-1} \int_{0}^{\infty}\left[\left(\rho^{s} u^{s}\right)(y+\alpha-\beta)-\left(\rho_{0} u_{0}\right)(y)\right] d y \\
& +\left(\frac{\gamma}{\gamma-1}-\frac{1}{2}\right) u_{-}^{2} \int_{0}^{\infty}\left[\rho^{s}(y+\alpha-\beta)-\rho_{0}(y)\right] d y \\
& -\int_{0}^{\infty}\left(J \psi-q^{s}\right)\left(-s_{3} \tau+\alpha-\beta\right) d \tau .
\end{aligned}
$$

Then it follows from direct calculations that,

$$
\begin{aligned}
I^{\prime}(\alpha)= & {\left[\rho_{+}\left(C_{v} \theta_{+}+\frac{u_{+}^{2}}{2}\right)-\rho^{s}\left(C_{v} \theta^{s}+\frac{\left(u^{s}\right)^{2}}{2}\right)(\alpha-\beta)\right] } \\
& -\frac{\gamma u_{-}}{\gamma-1}\left[\rho_{+} u_{+}-\left(\rho^{s} u^{s}\right)(\alpha-\beta)\right]+\left(\frac{\gamma}{\gamma-1}-\frac{1}{2}\right) u_{-}^{2}\left[\rho_{+}-\rho^{s}(\alpha-\beta)\right] \\
& -\frac{1}{s_{3}}\left[J\left(u_{-}-u^{s}\right)-q^{s}\right](\alpha-\beta) .
\end{aligned}
$$

In order to simplify and obtain a good and useful expression on $I^{\prime}(\alpha)$, let us integrate (2.4) over $[0, \xi]$ to get

$$
\left\{\begin{array}{l}
\rho^{s} u^{s}-\rho_{-} u_{-}=s_{3}\left(\rho^{s}-\rho_{-}\right), \\
p^{s}-p_{-}=s_{3}\left(\rho^{s} u^{s}-\rho_{-} u_{-}\right)-\left[\rho^{s}\left(u^{s}\right)^{2}-\rho_{-} u_{-}^{2}\right] \\
\left\{\rho_{ \pm}\left(e_{-}+\frac{u_{-}^{2}}{2}\right)-\rho^{s}\left[e^{s}+\frac{\left(u^{s}\right)^{2}}{2}\right]\right\}+\frac{1}{s_{3}} q^{s} \\
=\frac{1}{s_{3}}\left\{\rho_{-} u_{-}\left(e_{-}+\frac{u_{-}^{2}}{2}\right)-\rho^{s} u^{s}\left[e^{s}+\frac{\left(u^{s}\right)^{2}}{2}\right]+p_{-} u_{-}-p^{s} u^{s}\right\} .
\end{array}\right.
$$


Substituting (2.34) into (2.33), we further obtain

$$
\begin{aligned}
I^{\prime}(\alpha)= & {\left[\rho_{+}\left(C_{v} \theta_{+}+\frac{u_{+}^{2}}{2}\right)-\rho_{-}\left(C_{v} \theta_{-}+\frac{u_{-}^{2}}{2}\right)\right] } \\
& -\frac{\gamma u_{-}}{\gamma-1}\left(\rho_{+} u_{+}-\rho_{-} u_{-}\right)+\left(\frac{\gamma}{\gamma-1}-\frac{1}{2}\right) u_{-}^{2}\left(\rho_{+}-\rho_{-}\right) \\
& +\left\{\rho_{-}\left(C_{v} \theta_{-}+\frac{u_{-}^{2}}{2}\right)-\rho^{s}\left[C_{v} \theta^{s}+\frac{\left(u^{s}\right)^{2}}{2}\right](\alpha-\beta)\right\}+\frac{1}{s_{3}} q^{s}(\alpha-\beta) \\
& -\frac{\gamma u_{-}}{\gamma-1}\left[\rho_{-} u_{-}-\left(\rho^{s} u^{s}\right)(\alpha-\beta)\right]+\left(\frac{\gamma}{\gamma-1}-\frac{1}{2}\right) u_{-}^{2}\left[\rho_{-}-\rho^{s}(\alpha-\beta)\right] \\
& -\frac{1}{s_{3}}\left\{\left[\frac{1}{2} \rho^{s}\left(u^{s}\right)^{2}+\frac{\gamma p^{s}}{\gamma-1}+\left(\frac{1}{2}-\frac{\gamma}{\gamma-1}\right) \rho^{s} u^{s} u_{-}\right]\left(u_{-}-u^{s}\right)\right\}(\alpha-\beta),
\end{aligned}
$$

which is reduced to the following perfect equality by tedious and tricky calculations:

$$
\begin{aligned}
I^{\prime}(\alpha)= & {\left[\rho_{+}\left(C_{v} \theta_{+}+\frac{u_{+}^{2}}{2}\right)-\rho_{-}\left(C_{v} \theta_{-}+\frac{u_{-}^{2}}{2}\right)\right] } \\
& -\frac{\gamma u_{-}}{\gamma-1}\left(\rho_{+} u_{+}-\rho_{-} u_{-}\right)+\left(\frac{\gamma}{\gamma-1}-\frac{1}{2}\right) u_{-}^{2}\left(\rho_{+}-\rho_{-}\right):=m .
\end{aligned}
$$

Therefore, we get

$$
I(\alpha)-I(0)=m \alpha
$$

Hence, (2.30) holds if and only if

$$
\alpha=-\frac{I(0)}{m} .
$$

\subsection{Main theorem}

Now, we are ready to introduce the main results of this paper. Define

$$
\begin{aligned}
& \Phi_{0}(x)=-\int_{x}^{\infty}\left[\rho_{0}(y)-\rho^{s}(y, 0,0, \beta)\right] d y, \\
& \Psi_{0}(x)=-\int_{x}^{\infty}\left[\left(\rho_{0} u_{0}\right)(y)-\left(\rho^{s} u^{s}\right)(y, 0,0, \beta)\right] d y, \\
& W_{0}(x)=-\int_{x}^{\infty}\left[\rho_{0}\left(C_{v} \theta_{0}+\frac{u_{0}^{2}}{2}\right)(y)-\rho^{s}\left(C_{v} \theta^{s}+\frac{u^{s}}{2}\right)(y, 0,0, \beta)\right] d y .
\end{aligned}
$$

We derive the system satisfied by the anti-derivative unknowns $(\Phi, \Psi, W, \Gamma)$ from the definitions in (2.12) and (2.17) that 


$$
\left\{\begin{array}{l}
\Phi_{t}+u^{s} \Phi_{x}+\Psi_{x}+u_{x}^{s} \Phi=0, \\
\Psi_{t}+u^{s} \Psi_{x}+R \theta^{s} \Phi_{x}+R W_{x}+(\gamma-1) u_{x}^{s} \Psi-\frac{R \theta^{s}}{\rho^{s}} \rho_{x}^{s} \Phi=G_{1}, \\
C_{v} W_{t}+C_{v} u^{s} W_{x}+R \theta^{s} \Psi_{x}-\frac{q_{x}^{s}}{\rho^{s}} \Phi-\frac{p_{x}^{s}}{\rho^{s}} \Psi+\Gamma_{x}=G_{2}, \\
-\Gamma_{x x}+a \Gamma+\frac{4 b\left(\theta^{s}\right)^{3}}{\rho} W_{x}+\frac{4 b\left(\theta^{s}\right)^{3}}{\rho} \theta_{x}^{s} \Phi+\frac{4 b\left(\theta^{s}\right)^{3}}{C_{v} \rho} u_{x}^{s} \Psi=G_{3}
\end{array}\right.
$$

with initial data

$$
(\Phi, \Psi, W)(x, 0)=\left(\Phi_{0}, \Psi_{0}, W_{0}\right)(x),
$$

and the boundary condition obtained from (2.31) that

$$
W(0, t)-\frac{u_{-}}{R} \Psi(0, t)+\theta_{-} \Phi(0, t)=A(t)=O(1) \mathrm{e}^{-c \delta \beta} \mathrm{e}^{-c \delta t} .
$$

Hereinafter, $G_{i}(i=1,2,3)$ are expressed by

$$
\begin{aligned}
G_{1} & :=\frac{\gamma-1}{2} \rho \psi^{2}-u_{x}^{s} \Phi \psi-\psi \Psi_{x}=O(1)\left(|\psi|^{2}+\left|u_{x}^{s} \Phi\right|^{2}+\left|\Psi_{x}\right|^{2}\right), \\
G_{2} & :=-E^{s} \Phi_{x} \psi-u_{x}^{s} \psi \Psi-u^{s} u_{x}^{s} \psi \Phi-C_{v} \psi W_{x}-R \zeta \Psi_{x} \\
& =O(1)\left(\left|u_{x}^{s}\right||(\Phi, \Psi)||\psi|+\left|\left(\Phi_{x}, \Psi_{x}, W_{x}\right)\right||\psi|+\left|\zeta \Psi_{x}\right|\right), \\
G_{3} & :=q_{x}^{s}-b \zeta^{2}\left(\zeta^{2}+4 \theta^{s} \zeta+6\left(\theta^{s}\right)^{2}\right)+\frac{4 b\left(\theta^{s}\right)^{3}}{C_{v}} \psi^{2}=q_{x}^{s}+O(1)\left(|\psi|^{2}+|\zeta|^{2}\right) .
\end{aligned}
$$

Define the solution space $X_{m_{1}, m_{2}, M}(0, t)$ as

$$
\begin{aligned}
X_{m_{1}, m_{2}, M}(0, t):=\{ & (\Phi, \Psi, W, \Gamma) \mid(\Phi, \Psi, W) \in \sum_{k=0}^{3} C^{k}\left([0, t] ; H^{3-k}\left(\mathbb{R}_{+}\right)\right), \\
& \Gamma \in \sum_{k=0}^{4} C^{k}\left([0, t] ; H^{4-k}\left(\mathbb{R}_{+}\right)\right) \partial_{t}^{i}(\phi, \psi, \zeta) \in L^{2}\left(0, t ; H^{2-i}\left(\mathbb{R}_{+}\right)\right), \\
& \partial_{t}^{i} \omega \in L^{2}\left(0, t ; H^{3-i}\left(\mathbb{R}_{+}\right)\right)(i=0,1,2), \\
& \inf _{[0, t] \times \mathbb{R}_{+}} \rho(x, t) \geq m_{1}, \inf _{[0, t] \times \mathbb{R}_{+}} \theta(x, t) \geq m_{2}, \\
& \left.\sup _{[0, t] \times \mathbb{R}_{+}}\left(\sum_{k=0}^{3}\left\|\left(\partial_{t}^{k} \Phi, \partial_{t}^{k} \Psi\right)\right\|_{3-k}+\sum_{k=0}^{2}\left\|\partial_{t}^{k} W\right\|_{3-k}\right) \leq M\right\},
\end{aligned}
$$

where $m_{1}, m_{2}, M$ are positive constants and $\|\cdot\|_{k}:=\|\cdot\|_{H^{k}\left(\mathbb{R}_{+}\right)}$. Hereafter, we denote $\|\cdot\|:=\|\cdot\|_{0}$.

Then the main theorem of this paper is stated as follows. 
Theorem 2.1. Assume the adiabatic exponent $\gamma \in(1,2)$ and the states $\left(\rho_{+}, u_{+}, \theta_{+}\right)$and $\left(\rho_{-}, u_{-}, \theta_{-}\right)$are in $\widetilde{\Omega}_{\text {sub }}^{-}$with $u_{+}<u_{-}<0$ and $\left(\rho_{-}, u_{-}, \theta_{-}\right)$being at the 3-shock curve passing through $\left(\rho_{+}, u_{+}, \theta_{+}\right)$with shock speed $s_{3}$, i.e., (2.1)-(2.3) hold. If the initial data satisfies

$$
\begin{aligned}
& \left(\Phi_{0}, \Psi_{0}, W_{0}\right)(x) \in H^{3}\left(\mathbb{R}_{+}\right), \\
& \left(\rho_{0}, u_{0}, \theta_{0}\right)(x)-\left(\rho^{s}, u^{s}, \theta^{s}\right)(x, 0, \alpha, \beta) \in\left(H^{2} \cap L^{1}\right)\left(\mathbb{R}_{+}\right)
\end{aligned}
$$

and if there exist suitably small constants $\delta_{0}>0, \epsilon_{0}>0$ such that

$$
\delta \lesssim \delta_{0}, \quad\left\|\left(\Phi_{0}, \Psi_{0}, W_{0}\right)\right\|_{3}+\left\|\left(\phi_{t}, \psi_{t}, \zeta_{t}\right)(0)\right\|_{1}+\mathrm{e}^{-\delta \beta} \lesssim \epsilon_{0}
$$

then there exist positive constants $m_{1}, m_{2}$ and $M$ such that, the outflow problem (2.39)(2.41) admits a unique solution $(\Phi, \Psi, W, \Gamma)$, so that problem (1.1), (1.2) and (1.7) admits a unique solution $(\rho, u, \theta, q)(x, t)$, such that

$$
(\Phi, \Psi, W, \Gamma) \in X_{m_{1}, m_{2}, M}(0,+\infty) .
$$

Here $\left(\rho^{s}, u^{s}, \theta^{s}, q^{s}\right)(t, x)$ is the shock wave defined in (2.4). Furthermore, it holds

$$
\sup _{x \geq 0}\left|(\rho, u, \theta, q)(t, x)-\left(\rho^{s}, u^{s}, \theta^{s}, q^{s}\right)(x, t, \alpha, \beta)\right| \rightarrow 0 \quad \text { as } \quad t \rightarrow+\infty,
$$

where $\alpha=\alpha(\beta)$ is defined in (2.34).

We will employ the anti-derivative unknowns $(\Phi, \Psi, W, \Gamma)$ to show the main theorem by presenting two propositions concerned with local existence and a priori estimates respectively. First, the local existence of the solution to system (2.39)(2.41) is stated as follows.

Proposition 2.1 (Local existence). Under the conditions of Theorem 2.1, there exist positive constants $\bar{\delta}_{2}, \bar{\epsilon}_{2}$ and $\bar{C}\left(\bar{C}_{\bar{\epsilon}} \leq \epsilon_{2}\right)$ such that the following statements hold: For any $\tau>0$, let

$$
\left(\Phi_{\tau}, \Psi_{\tau}, W_{\tau}\right)(x):=(\Phi, \Psi, W)(x, \tau) \in H^{3}\left(\mathbb{R}_{+}\right)
$$

for any $M \in\left(0, \bar{\epsilon}_{2}\right), \delta \leq \bar{\delta}_{2}(<\bar{\delta})$ and $\beta>1$. Assume that

$$
\left\|\left(\Phi_{\tau}, \Psi_{\tau}, W_{\tau}\right)\right\|_{3} \lesssim M, \quad \sup _{t \geq 0}\left\{|A(t)|+\left|A^{\prime}(t)\right|\right\} \lesssim \mathrm{e}^{-\delta \beta},
$$

then there exists a positive constant $t_{0}=t_{0}(M, \beta)$ independent on $\tau$ such that, problem (2.39), (2.41) and (2.47) admits a unique solution $(\Phi, \Psi, W, \Gamma)(x, t) \in \mathbb{X}_{m_{1}, m_{2}, M}\left(\tau, \tau+t_{0}\right)$. 
The proof of Proposition 2.1 is standard. Actually, one can follow the argument in the proof of [5, Proposition 3.1, pp.601-603] to show Proposition 2.1 similarly. Therefore, we omit the proof for the shortness. Based on Proposition 2.1, we can show Theorem 2.1 if the following proposition is proved.

Proposition 2.2 (A priori estimate). Under the conditions of Proposition 2.1, there are constants $\bar{\delta}_{3}\left(\leq \bar{\delta}_{2}\right)$ and $\bar{\epsilon}_{3}\left(\leq \bar{\epsilon}_{2}\right)$ such that, if $(\Phi, \Psi, W, \Gamma)(x, t) \in \mathbb{X}_{m_{1}, m_{2}, M}(0, T)$ for some $T>0$ is a solution to the initial-boundary value problem (2.39)-(2.41). If $\delta \leq \bar{\delta}_{3}$ and for $t \in[0, T]$

$$
\sup _{\tau \in[0, t]}\left\{\|(\Phi, \Psi, W, \Gamma)(\tau)\|_{3}+\left\|\left(\phi_{t}, \psi_{t}, \zeta_{t}, \omega_{t}\right)(\tau)\right\|_{1}\right\} \leq \bar{\epsilon}_{3}
$$

then it holds that for $t \in[0, T]$,

$$
\begin{aligned}
& \|(\Phi, \Psi, W, \Gamma)(t)\|^{2}+\|(\phi, \psi, \zeta)(t)\|_{2}^{2}+\left\|\left(\phi_{t}, \psi_{t}, \zeta_{t}\right)(t)\right\|_{1}^{2} \\
& \quad+\left\|\left(\phi_{t t}, \psi_{t t}, \zeta_{t t}\right)(t)\right\|^{2}+\|\omega(t)\|_{3}^{2}+\left\|\omega_{t}(t)\right\|_{2}^{2} \\
& \quad+\int_{0}^{t}|(\Phi, \Psi, W)|^{2}(0, \tau) d \tau+\int_{0}^{t} \int_{\mathbb{R}_{+}}\left(\left|u_{x}^{s} \|(\Psi, W)\right|^{2}+\Gamma^{2}\right) d x d \tau \\
& \quad+\int_{0}^{t}\left(\|(\phi, \psi, \zeta)(\tau)\|_{2}^{2}+\|w(\tau)\|_{3}^{2}+\left\|w_{t}(\tau)\right\|_{2}^{2}\right) d \tau \\
& \quad+\int_{0}^{t}\left|\left(\phi, \phi_{x}, \psi_{x}, \zeta_{x}, w_{x}, \phi_{x x}, \psi_{x x}, \zeta_{x x}, w_{x x}, w_{x x x}, \phi_{t x}, \psi_{t x}, \zeta_{t x}, w_{t x}\right)\right|^{2}(0, \tau) d \tau \\
& \lesssim\left\|\left(\Phi_{0}, \Psi_{0}, W_{0}\right)\right\|^{2}+\left\|\left(\phi_{0}, \psi_{0}, \zeta_{0}\right)\right\|_{1}^{2}+\left\|\left(\phi_{t}, \psi_{t}, \zeta_{t}\right)(0)\right\|_{1}^{2}+\left\|\left(\phi_{t t}, \psi_{t t}, \zeta_{t t}\right)(0)\right\|^{2}+\mathrm{e}^{-\delta \beta}
\end{aligned}
$$

The a priori estimate (2.48) will be shown in the rest sections. Actually, once Proposition 2.2 is obtained, the local solution $(\phi, \psi, \zeta, \omega)(x, t)$ obtained in Proposition 2.1 can be extended to $t=\infty$. Moreover, estimate (2.48) implies that

$$
\int_{0}^{\infty}\left(\|(\phi, \psi, \zeta, \omega)(\tau)\|^{2}+\frac{d}{d t}\|(\phi, \psi, \zeta, \omega)(\tau)\|^{2}\right) d \tau<+\infty,
$$

which together with the Sobolev inequality implies the asymptotic behavior (2.46). This concludes the proof of Theorem 2.1.

\section{Basic energy estimate on fluid dynamic perturbations}

In this and next two sections, we focus on proving Proposition 2.2. Firstly, we prove basic energy estimates. Set 


$$
N(t):=\sup _{\tau \in[0, t]}\left\{\|(\Phi, \Psi, W, \Gamma)(\tau)\|_{3}+\left\|\left(\phi_{t}, \psi_{t}, \zeta_{t}, \omega_{t}\right)(\tau)\right\|_{1}\right\}
$$

For system (2.14) with (2.15) and (2.16), we get

Lemma 3.1. Under the assumption of Proposition 2.2, if $N(t)$ and $\delta$ are suitably small, it holds that

$$
\begin{aligned}
& \|(\phi, \psi, \zeta)(t)\|^{2}+\int_{0}^{t}\left(\phi^{2}+\zeta^{2}\right)(0, \tau) d \tau+\left\|\left(\omega, \omega_{x}\right)(\tau)\right\|^{2} d \tau \\
\lesssim & \left\|\left(\phi_{0}, \psi_{0}, \zeta_{0}\right)\right\|^{2}+\mathrm{e}^{-\delta \beta}+(\delta+N(t)) \int_{0}^{t}\left\|\left(\phi, \psi, \zeta, \phi_{x}, \psi_{x}, \zeta_{x}, \zeta_{x x}, \omega_{x x}\right)(\tau)\right\|^{2} d \tau
\end{aligned}
$$

Proof. Multiplying the equations in (2.14) by $\frac{R \theta}{\rho} \phi, \rho \psi, \frac{\rho \zeta}{\theta}, \frac{R \omega}{4 b \theta^{4}}$ respectively, we have

$$
\begin{aligned}
& \left(\frac{R \theta}{2 \rho} \phi^{2}\right)_{t}+\left(\frac{R \theta u}{2 \rho} \phi^{2}\right)_{x}+R \theta \phi \psi_{x}=\left\{\left(\frac{R \theta}{2 \rho}\right)_{t}+\left(\frac{R \theta u}{2 \rho}\right)_{x}\right\} \phi^{2}+\frac{R \theta}{\rho} \phi Q_{1}, \\
& \left(\frac{\rho \psi^{2}}{2}\right)_{t}+\left(\frac{\rho u \psi^{2}}{2}\right)_{x}+R \rho \psi \zeta_{x}+R \theta \phi_{x} \psi=\left\{\left(\frac{\rho}{2}\right)_{t}+\left(\frac{\rho u}{2}\right)_{x}\right\} \psi^{2}+\rho \psi Q_{2}, \\
& \left(\frac{C_{v} \rho}{2 \theta} \zeta^{2}\right)_{t}+\left(\frac{C_{v} \rho u}{2 \theta} \zeta^{2}\right)_{x}+R \rho \zeta \psi_{x}+\frac{\rho \zeta}{\theta} \omega_{x} \\
= & \left\{\left(\frac{C_{v} \rho}{2 \theta}\right)_{t}+\left(\frac{C_{v} \rho u}{2 \theta}\right)_{x}\right\} \zeta^{2}+\frac{\rho \zeta}{\theta} Q_{3}, \\
& -\left(\frac{R \omega \omega_{x}}{4 b \theta^{4}}\right)_{x}+\left(\frac{R}{4 b \theta^{4}}\right)_{x} \omega \omega_{x}+\frac{R \omega_{x}^{2}}{4 b \theta^{4}}+\frac{a R \omega^{2}}{4 b \theta^{4}}+\frac{\rho \zeta_{x}}{\theta} \omega \\
= & -\frac{R \theta_{x}^{s} \zeta \omega}{\theta^{4}}\left\{\theta^{2}+\theta \theta^{s}+\left(\theta^{s}\right)^{2}\right\}+\frac{R \omega q_{x x}^{s}}{4 b \theta^{4}} .
\end{aligned}
$$

Summing them up, we have

$$
\left(\frac{R \theta}{2 \rho} \phi^{2}+\frac{\rho \psi^{2}}{2}+\frac{C_{v} \rho}{2 \theta} \zeta^{2}\right)_{t}+\left(\frac{R}{4 b \theta^{4}}\right)_{x} \omega \omega_{x}+\frac{R \omega_{x}^{2}}{4 b \theta^{4}}+\frac{a R \omega^{2}}{4 b \theta^{4}}+I_{1 x}=I_{2}
$$

where

$$
\begin{aligned}
& I_{1}:=\frac{R \theta u}{2 \rho} \phi^{2}+\frac{\rho u \psi^{2}}{2}+\frac{C_{v} \rho u \zeta^{2}}{2 \theta}+R \rho \psi \zeta+R \theta \phi \psi-\frac{R \omega \omega_{x}}{4 b \theta^{4}}+\frac{\rho \zeta}{\theta} \omega, \\
& I_{2}:=\left\{\left(\frac{R \theta}{2 \rho}\right)_{t}+\left(\frac{R \theta u}{2 \rho}\right)_{x}\right\} \phi^{2}+\left\{\left(\frac{C_{v} \rho}{2 \theta}\right)_{t}+\left(\frac{C_{v} \rho u}{2 \theta}\right)_{x}\right\} \zeta^{2}+R \rho_{x} \psi \zeta
\end{aligned}
$$




$$
\begin{aligned}
& +R \theta_{x} \phi \psi+\left(\frac{\rho}{\theta}\right)_{x} \zeta \omega+\frac{R \theta}{\rho} \phi Q_{1}+\rho \psi Q_{2}+\frac{\rho \zeta}{\theta} Q_{3} \\
& -\frac{R \theta_{x}^{s} \zeta \omega}{\theta^{4}}\left\{\theta^{2}+\theta \theta^{s}+\left(\theta^{s}\right)^{2}\right\}+\frac{R \omega q_{x x}^{s}}{4 b \theta^{4}} .
\end{aligned}
$$

It is easy to see that

$$
\left|I_{2}\right| \lesssim(\delta+N(t))\left|\left(\phi, \psi, \zeta, \phi_{x}, \psi_{x}, \zeta_{x}, \zeta_{x x}\right)\right|^{2}+\left|\omega q_{x x}^{s}\right|
$$

Moreover, it follows from the boundary condition $u(0, t)=u_{-}<0$ that

$$
\begin{aligned}
-I_{1}(0, t) & =-\left(\frac{R \theta u}{2 \rho} \phi^{2}+\frac{\rho u \psi^{2}}{2}+\frac{C_{v} \rho u \zeta^{2}}{2 \theta}+R \rho \psi \zeta+R \theta \phi \psi-\frac{R \omega \omega_{x}}{4 b \theta^{4}}+\frac{\rho \zeta}{\theta} \omega\right)(0, t) \\
& \geq c\left(\phi^{2}+\zeta^{2}\right)(0, t)-C\left(\psi^{2}+\omega^{2}+\omega \omega_{x}\right)(0, t) \\
& \geq c\left(\phi^{2}+\zeta^{2}\right)(0, t)-C \delta \mathrm{e}^{-c \delta \beta} \mathrm{e}^{-c \delta t}
\end{aligned}
$$

where positive constants $c$ and $C$ do not depend on $(\phi, \psi, \zeta, \omega)$. Integrating (3.7) over $\mathbb{R}_{+} \times[0, t]$ and using (3.9)-(3.10), we get (3.2). This completes the proof of Lemma 3.1.

Next, in order to estimate $\int_{0}^{t}\|(\phi, \psi, \zeta)(\tau)\|^{2} d \tau$, we will derive the following estimate on the anti-derivative functions $(\Phi, \Psi, W, \Gamma)$.

Lemma 3.2. Under the assumptions listed in Proposition 2.2, if $N(t)$ and $\delta$ are suitably small, and if $\gamma \in(1,2)$, it holds that

$$
\begin{aligned}
\|(\Phi, \Psi, W)(t)\|^{2}+\int_{0}^{t}|(\Psi, \Psi, W)|^{2}(0, \tau) d \tau \\
\quad+\int_{0}^{t} \int_{\mathbb{R}_{+}}\left(\left|u_{x}^{s} \|(\Psi, W)\right|^{2}+\Gamma^{2}+\Gamma_{x}^{2}\right)(x, \tau) d x d \tau \\
\lesssim\left\|\left(\Phi_{0}, \Psi_{0}, W_{0}\right)\right\|^{2}+\left\|\left(\phi_{0}, \psi_{0}, \zeta_{0}\right)\right\|^{2}+\mathrm{e}^{-\delta \beta} \\
\quad+(\delta+N(t)) \int_{0}^{t}\left\|\left(\phi, \psi, \zeta, \phi_{x}, \psi_{x}, \zeta_{x}, \zeta_{x x}, \omega_{x x}\right)(\tau)\right\|^{2} d \tau .
\end{aligned}
$$

Proof. Multiplying the first three equations in (2.39) by $\frac{1}{\rho^{s}} \Phi, \frac{\Psi}{p^{s}}$ and $\frac{W}{\theta^{s} p^{s}}$ respectively, we obtain the identities

$$
\left(\frac{\Phi^{2}}{2 \rho^{s}}\right)_{t}+\left(\frac{u^{s}}{2 \rho^{s}} \Phi^{2}\right)_{x}+\frac{1}{\rho^{s}} \Phi \Psi_{x}=0
$$




$$
\begin{aligned}
& \left(\frac{\Psi^{2}}{2 p^{s}}\right)_{t}+\left(\frac{u^{s}}{2 p^{s}} \Psi^{2}\right)_{x}+\left(\frac{1}{\rho^{s}} \Phi\right)_{x} \Psi+\frac{R \Psi}{p^{s}} W_{x} \\
& \quad+\left(\frac{(\gamma-2) u_{x}^{s}}{2 p^{s}}-\frac{(\gamma-1) q_{x}^{s}}{2\left(p^{s}\right)^{2}}\right) \Psi^{2}=\frac{\Psi G_{1}}{p^{s}} \\
& \left(\frac{C_{v} W^{2}}{2 p^{s} \theta^{s}}\right)_{t}+\left(\frac{C_{v} u^{s} W^{2}}{2 p^{s} \theta^{s}}\right)_{x}+\left(\frac{R}{p^{s}} \Psi\right)_{x} W+\frac{W \Gamma_{x}}{\theta^{s} p^{s}} \\
& \quad+\frac{C_{v} \gamma\left(-u_{x}^{s}\right)}{\theta^{s} p^{s}} W^{2}-\frac{R q_{x}^{s}}{\theta^{s}\left(p^{s}\right)^{2}} W^{2}-\frac{R q_{x}^{s}}{\left(p^{s}\right)^{2}} \Psi W=\frac{W G_{2}}{\theta^{s} p^{s}} .
\end{aligned}
$$

Summing (3.12)-(3.14) together, one has

$$
\begin{aligned}
& \left(\frac{\Phi^{2}}{2 \rho^{s}}+\frac{\Psi^{2}}{2 p^{s}}+\frac{C_{v} W^{2}}{2 p^{s} \theta^{s}}\right)_{t}+I_{3 x}+\frac{(\gamma-2) u_{x}^{s}}{2 p^{s}} \Psi^{2}+\frac{C_{v} \gamma\left(-u_{x}^{s}\right)}{\theta^{s} p^{s}} W^{2}+\frac{W \Gamma_{x}}{\theta^{s} p^{s}} \\
= & \frac{\Psi G_{1}}{\rho^{s}}+\frac{W G_{2}}{\theta^{s} p^{s}}+\frac{(\gamma-1) q_{x}^{s}}{2\left(p^{s}\right)^{2}} \Psi^{2}+\frac{R q_{x}^{s}}{\theta^{s}\left(p^{s}\right)^{2}} W^{2}+\frac{R q_{x}^{s}}{\left(p^{s}\right)^{2}} \Psi W,
\end{aligned}
$$

where

$$
I_{3}:=\frac{u^{s} \Phi^{2}}{2 \rho^{s}}+\frac{u^{s} \Psi^{2}}{2 p^{s}}+\frac{C_{v} u^{s} W^{2}}{2 \theta^{s} p^{s}}+\frac{\Phi \Psi}{\rho^{s}}+\frac{R}{p^{s}} \Psi W .
$$

Finally, multiplying $(2.39 \mathrm{~d})$ by $\frac{\rho}{4 b\left(\theta^{s}\right)^{2}} \frac{\Gamma}{\theta^{s} p^{s}}$ yields

$$
\begin{aligned}
& -\left[\frac{\rho}{4 b\left(\theta^{s}\right)^{3}} \frac{\Gamma \Gamma_{x}}{p^{s}}\right]_{x}+\left[\frac{\rho}{4 b\left(\theta^{s}\right)^{3} p^{s}}\right]_{x} \Gamma \Gamma_{x}+\frac{\rho\left(\Gamma_{x}^{2}+a \Gamma^{2}\right)}{4 b\left(\theta^{s}\right)^{3} p^{s}} \\
& +\frac{\Gamma W_{x}}{\theta^{s} p^{s}}+\frac{\theta_{x}^{s} \Phi \Gamma}{\theta^{s} p^{s}}+\frac{u_{x}^{s} \Psi \Gamma}{C_{v} \theta^{s} p^{s}}=\frac{\rho \Gamma G_{3}}{4 b\left(\theta^{s}\right)^{3} p^{s}} .
\end{aligned}
$$

Then it follows from (3.15) and (3.17) that

$$
\begin{aligned}
& \left(\frac{\Phi^{2}}{2 \rho^{s}}+\frac{\Psi^{2}}{2 p^{s}}+\frac{C_{v} W^{2}}{2 p^{s} \theta^{s}}\right)_{t}+\frac{\rho\left(\Gamma_{x}^{2}+a \Gamma^{2}\right)}{4 b\left(\theta^{s}\right)^{3} p^{s}} \\
& +\left[I_{3}-\frac{\rho \Gamma \Gamma_{x}}{4 b\left(\theta^{s}\right)^{3} p^{s}}+\frac{W \Gamma}{\theta^{s} p^{s}}\right]_{x}+\left[\frac{\rho}{4 b\left(\theta^{s}\right)^{3} p^{s}}\right]_{x} \Gamma \Gamma_{x}-\left[\frac{1}{\theta^{s} p^{s}}\right]_{x} W \Gamma \\
& \quad+\frac{(\gamma-2) u_{x}^{s}}{2 p^{s}} \Psi^{2}+\frac{C_{v} \gamma\left(-u_{x}^{s}\right)}{\theta^{s} p^{s}} W^{2}+\frac{\theta_{x}^{s} \Phi \Gamma}{\theta^{s} p^{s}}+\frac{u_{x}^{s} \Psi \Gamma}{C_{v} \theta^{s} p^{s}} \\
& =O(1)\left|\Psi G_{1}+W G_{2}+\Gamma G_{3}\right|+O(1)\left|q_{x}^{s}\right|\left|\Psi^{2}+W^{2}+\Psi W\right| .
\end{aligned}
$$


Note that by boundary condition (2.41), we have

$$
\Psi(0, t)=\frac{R}{u_{-}}\left(W+\theta_{-} \Phi\right)(0, t)+O(1) \mathrm{e}^{-c \delta \beta} \mathrm{e}^{-c \delta t} .
$$

So

$$
\begin{aligned}
-I_{3}(0, t)= & \left(\frac{-u_{-} \Phi^{2}}{2 \rho_{-}}+\frac{\left(-u_{-}\right) \Psi^{2}}{2 p_{-}}+\frac{C_{v}\left(-u_{-}\right) W^{2}}{2 p_{-} \theta_{-}}\right)(0, t) \\
& -\frac{\Psi}{\rho_{-} \theta_{-}}\left(\theta_{-} \Phi+W\right)(0, t)+O(1) \mathrm{e}^{-c \delta \beta} \mathrm{e}^{-c \delta t} \\
= & \frac{-u_{-}}{p_{-}}\left(\frac{\Phi^{2}}{2}+\frac{\Psi^{2}}{2}+\frac{C_{v} W^{2}}{2 \theta_{-}}\right)(0, t)-\frac{\Psi}{\theta_{-} p_{-}} \frac{u_{-}}{R} \Psi(0, t)+O(1) \mathrm{e}^{-c \delta \beta} \mathrm{e}^{-c \delta t} \\
= & \frac{\left(-u_{-}\right)}{p_{-}}\left(\frac{R \theta_{-} \Phi^{2}}{2}+\frac{3 \Psi^{2}}{2}+\frac{C_{v} W^{2}}{2 \theta_{-}}\right)(0, t)+O(1) \mathrm{e}^{-c \delta \beta} \mathrm{e}^{-c \delta t}
\end{aligned}
$$

So (3.19) implies that the boundary term $-I_{3}(0, t)$ can be controlled.

Suppose $1<\gamma<2$, then $\gamma-2<0$. Then integrating (3.18) over $\mathbb{R}_{+} \times(0, t)$, integrating by part, and using (3.19) and the Sobolev inequality, we obtain that

$$
\begin{aligned}
& \|(\Phi, \Psi, W)(t)\|^{2}+\int_{0}^{t}\left(|(\Phi, \Psi, W)|^{2}(0, \tau)+\|\Gamma(\tau)\|_{1}^{2}\right) d \tau \\
& +\int_{0}^{t} \int_{\mathbb{R}_{+}}\left|u_{x}^{s}\right|\left(\Psi^{2}+W^{2}\right) d x d \tau \\
\lesssim & \left\|\left(\Phi_{0}, \Psi_{0}, W_{0}\right)\right\|^{2}+\int_{0}^{t}\left|\left(\Gamma \Gamma_{x}+W \Gamma\right)\right|(0, \tau) d \tau \\
& +\int_{0}^{t} \int_{\mathbb{R}_{+}}\left|\left(u_{x}^{s}, \theta_{x}^{s}\right)\right|\left|\Gamma\left(\Gamma_{x}+\Phi+\Psi+W\right)\right| d x d \tau+\delta \int_{0}^{t} \mathrm{e}^{-c \delta \beta} \mathrm{e}^{-c \delta \tau} d \tau \\
& +\int_{0}^{t} \int_{\mathbb{R}_{+}}\left(\left|q_{x}^{s} \| \Psi^{2}+W^{2}+\Psi W\right|+\left|\Psi G_{1}+W G_{2}+\Gamma G_{3}\right|\right) d x d \tau .
\end{aligned}
$$

Note that

$$
q^{s}=-\frac{4 b}{a}\left(\theta^{s}\right)^{3} \theta_{x}^{s}, \quad \Gamma_{x}(0, t)=-q^{s}(0, t)=O(1)\left|\theta_{x}^{s}(0, t)\right|, \quad \Gamma_{x}=\omega .
$$

So we have

$$
\begin{aligned}
\int_{0}^{t} \Gamma^{2}(0, \tau) d \tau & \lesssim \int_{0}^{t}\|\Gamma(\tau)\|_{\infty}^{2} d \tau \lesssim \int_{0}^{t}\|\Gamma(\tau)\|\left\|\Gamma_{x}(\tau)\right\| d \tau \\
& \lesssim \frac{1}{4} \int_{0}^{t}\|\Gamma(\tau)\|^{2} d \tau+\int_{0}^{t}\|\omega(\tau)\|^{2} d \tau
\end{aligned}
$$


and then

$$
\begin{aligned}
& \int_{0}^{t} \int_{0}^{t}\left|\left(\Gamma \Gamma_{x}+W \Gamma\right)\right|(0, \tau) d \tau \\
\lesssim & \frac{1}{4} \int_{0}^{t} W^{2}(0, \tau) d \tau+\int_{0}^{t} \Gamma_{x}^{2}(0, \tau) d \tau+\int_{0}^{t} \Gamma^{2}(0, \tau) d \tau \\
\lesssim & \frac{1}{4} \int_{0}^{t} W^{2}(0, \tau) d \tau+\int_{0}^{t}\left(\theta_{x}^{s}\right)^{2}(0, t)(0, \tau) d \tau+\int_{0}^{t} \Gamma^{2}(0, \tau) d \tau \\
\lesssim & \frac{1}{4} \int_{0}^{t}\left(W^{2}(0, \tau)+\|\Gamma(\tau)\|^{2}\right) d \tau+\int_{0}^{t}\|\omega(\tau)\|^{2} d \tau+\mathrm{e}^{-\delta \beta} .
\end{aligned}
$$

Therefore, it follows from (3.20) that

$$
\begin{aligned}
& \|(\Phi, \Psi, W)(t) \|^{2}+\int_{0}^{t}\left(|(\Phi, \Psi, W)|^{2}(0, \tau)+\|\Gamma(\tau)\|_{1}^{2}\right) d \tau \\
&+\int_{0}^{t} \int_{\mathbb{R}_{+}}\left|u_{x}^{s}\right|\left(\Psi^{2}+W^{2}\right) d x d \tau \\
& \lesssim\left\|\left(\Phi_{0}, \Psi_{0}, W_{0}\right)\right\|^{2}+\mathrm{e}^{-\delta \beta}+\int_{0}^{t}\|\omega(\tau)\|^{2} d \tau \\
& \quad+\int_{0}^{t} \int_{\mathbb{R}_{+}}\left|\Psi G_{1}+W G_{2}+\Gamma G_{3}\right| d x d \tau+\int_{0}^{t} \int_{\mathbb{R}_{+}}\left|\theta_{x}^{s}\right|^{2} d x d \tau .
\end{aligned}
$$

Finally, the last two terms on the right-hand side of (3.24) can be estimated as follows:

$$
\begin{aligned}
& \int_{0}^{t} \int_{\mathbb{R}_{+}}\left|\Psi G_{1}+W G_{2}+\Gamma G_{3}\right| d x d \tau \\
\lesssim & (\delta+N(t)) \int_{0}^{t} \int_{\mathbb{R}_{+}}\left|u_{x}^{s}\right||(\Psi, W)|^{2} d x d \tau \\
& +N(t) \int_{0}^{t} \int_{\mathbb{R}_{+}}|(\psi, \psi, \zeta)(\tau)|^{2} d x d \tau+\int_{0}^{t} \int_{\mathbb{R}_{+}}\left|\theta_{x}^{s}\right|^{2} d x d \tau
\end{aligned}
$$

and

$$
\int_{0}^{t} \int_{\mathbb{R}_{+}}\left|\theta_{x}^{s}\right|^{2} d x d \tau \lesssim \delta^{2} \mathrm{e}^{-\delta \beta} \int_{0}^{t} \int_{\mathbb{R}_{+}} \mathrm{e}^{-c \delta \xi} \mathrm{e}^{-c \delta \tau} d \xi d \tau \lesssim \mathrm{e}^{-\delta \beta}
$$

Therefore, substituting (3.24), (3.25) into (3.23) and using (3.2), we can get (3.11). This completes the proof of Lemma 3.2.

Remark 3.1. We see that from the last equation of (2.39)

$$
w_{x}=-q^{s}+a \Gamma+b \zeta\left(\theta+\theta^{s}\right)\left(\theta^{2}+\theta \theta^{s}+\left(\theta^{s}\right)^{2}\right) .
$$


By applying Lemmas 3.1 and 3.2, we can obtain the following estimate of $w_{x}$ on the boundary

$$
\begin{aligned}
& \quad \int_{0}^{t} w_{x}^{2}(0, \tau) d \tau \lesssim \int_{0}^{t}\left(q_{x}^{s}\right)^{2}(0, \tau) d \tau+\int_{0}^{t}\left(\Gamma^{2}+\zeta^{2}\right)(0, \tau) d \tau \\
& \lesssim\left\|\left(\Phi_{0}, \Psi_{0}, W_{0}\right)\right\|^{2}+\left\|\left(\phi_{0}, \psi_{0}, \zeta_{0}\right)\right\|^{2}+\mathrm{e}^{-\delta \beta} \\
& \quad+(\delta+N(t)) \int_{0}^{t}\left\|\left(\phi, \psi, \zeta, \phi_{x}, \psi_{x}, \zeta_{x}, \zeta_{x x}\right)(\tau)\right\|^{2} d \tau .
\end{aligned}
$$

\section{High-order energy estimates on fluid dynamic perturbations}

In this section, we will focus on establishing the high-order energy estimates in two steps. Firstly, the first-order energy estimates are established in Subsection 4.1. Then the second-order energy estimates are established in Subsection 4.2.

\subsection{First-order energy estimates}

To establish the first-order energy estimate, we will first establish the following estimate on the time derivatives.

Lemma 4.1. Under the assumption of Proposition 2.2, if $N(t)$ and $\delta$ are suitably small, it holds that

$$
\begin{gathered}
\left\|\left(\phi_{t}, \psi_{t}, \zeta_{t}\right)(t)\right\|^{2}+\int_{0}^{t}\left(\phi_{t}^{2}+\zeta_{t}^{2}\right)(0, \tau)+\left\|\left(\omega_{t}, \omega_{t x}\right)(\tau)\right\|^{2} d \tau \\
\lesssim\left\|\left(\phi_{0}, \psi_{0}, \zeta_{0}\right)\right\|^{2}+\left\|\left(\phi_{t}, \psi_{t}, \zeta_{t}\right)(0)\right\|^{2}+\mathrm{e}^{-\delta \beta} \\
+(\delta+N(t)) \int_{0}^{t}\left(\|(\phi, \psi, \zeta)(\tau)\|_{2}^{2}+\left\|\omega_{t x x}(\tau)\right\|^{2}\right) d \tau .
\end{gathered}
$$

Proof. Differentiating each equation in (2.14) with respect to $t$, then multiplying them by $\frac{R \theta}{\rho} \phi_{t}, \rho \psi_{t}, \frac{\rho}{\theta} \zeta_{t}$ and $\frac{\rho}{4 b \theta^{4}} w_{t}$ respectively, and then adding all the resulting equations together, we have

$$
\left(\frac{R \theta}{2 \rho} \phi_{t}^{2}+\frac{\rho}{2} \psi_{t}^{2}+\frac{C_{v} \rho}{2 \theta} \zeta_{t}^{2}\right)_{t}+\frac{\rho w_{x t}^{2}}{4 b \theta^{4}}+\left(\frac{\rho}{4 b \theta^{4}}\right)_{x} w_{t} w_{x t}+\frac{a \rho w_{t}^{2}}{4 b \theta^{4}}+I_{4 x}=I_{5},
$$

where

$$
I_{4}:=\frac{R \theta u}{2 \rho} \phi_{t}^{2}+\frac{\rho u}{2} \psi_{t}^{2}+\frac{C_{v} \rho u}{2 \theta} \zeta_{t}^{2}+R \theta \phi_{t} \psi_{t}+R \rho \zeta_{t} \psi_{t}+\frac{\rho}{\theta} \zeta_{t} \omega_{t}-\frac{\rho w_{t}}{4 b \theta^{4}} w_{x t}
$$




$$
\begin{aligned}
I_{5} & :=\left\{\left(\frac{R \theta}{2 \rho}\right)_{t}+\left(\frac{R \theta u}{2 \rho}\right)_{x}\right\} \phi_{t}^{2}+\left\{\left(\frac{C_{v} \rho}{2 \theta}\right)_{t}+\left(\frac{C_{v} \rho u}{2 \theta}\right)_{x}\right\} \zeta_{t}^{2}+R \theta_{x} \phi_{t} \psi_{t} \\
& +R \rho_{x} \zeta_{t} \psi_{t}+\left(Q_{1 t}-\rho_{t} \psi_{x}-u_{t} \phi_{x}\right) \frac{R \theta}{\rho} \phi_{t}+\left[Q_{2 t}-u_{t} \psi_{x}-\left(\frac{R \theta}{\rho}\right)_{t} \phi_{x}\right] \rho \psi_{t} \\
& +\left(Q_{3 t}-C_{v} u_{t} \zeta_{x}-R \theta_{t} \psi_{x}\right) \frac{\rho}{\theta} \zeta_{t}+\left(\frac{\rho}{\theta}\right)_{x} \zeta_{t} \omega_{t} \\
& +\left\{q_{t x x}^{s}-12 b \theta^{2} \theta_{t} \zeta_{x}-\left[4 b \theta_{x}^{s} \zeta\left(\theta^{2}+\theta \theta^{s}+\left(\theta^{s}\right)^{2}\right)\right]_{t}\right\} \frac{w_{t}}{4 b \theta^{4}} .
\end{aligned}
$$

For the boundary term $I_{4}$, we note that

$$
\begin{aligned}
-I_{4}(0, t) & =-\left(\frac{R \theta u}{2 \rho} \phi_{t}^{2}+\frac{\rho u}{2} \psi_{t}^{2}+\frac{C_{v} \rho u}{2 \theta} \zeta_{t}^{2}+R \theta \phi_{t} \psi_{t}+R \rho \zeta_{t} \psi_{t}+\frac{\rho}{\theta} \zeta_{t} \omega_{t}-\frac{\rho w_{t}}{4 b \theta^{4}} w_{x t}\right)(0, t) \\
& \geq c\left(\phi_{t}^{2}+\zeta_{t}^{2}\right)(0, t)-C\left(\psi_{t}^{2}+\omega_{t}^{2}+\omega_{t} \omega_{t x}\right)(0, t) \\
& \geq c\left(\phi_{t}^{2}+\zeta_{t}^{2}\right)(0, t)-C \delta \mathrm{e}^{-c \delta \beta} \mathrm{e}^{-c \delta t}
\end{aligned}
$$

Then integrating (4.2) over $\mathbb{R}_{+} \times[0, t]$ and using (4.5), we obtain

$$
\begin{aligned}
& \quad\left\|\left(\phi_{t}, \psi_{t}, \zeta_{t}\right)(t)\right\|^{2}+\int_{0}^{t}\left(\left(\phi_{t}^{2}+\zeta_{t}^{2}\right)(0, \tau)+\left\|\left(\omega_{t}, \omega_{t x}\right)(\tau)\right\|^{2}\right) d \tau \\
& \lesssim\left\|\left(\phi_{t}, \psi_{t}, \zeta_{t}\right)(0)\right\|^{2}+\mathrm{e}^{-c \delta \beta}+\int_{0}^{t}\left(\psi_{t}^{2}+\omega_{t}^{2}+\left|\omega_{t x} \omega_{t}\right|\right)(0, \tau) d \tau \\
& \quad+(\delta+N(t)) \int_{0}^{t} \int_{\mathbb{R}^{+}}\left|\left(\phi, \psi, \zeta, \phi_{x}, \psi_{x}, \zeta_{x}, \zeta_{x x}\right)\right|^{2}(x, \tau) d x d \tau \\
& \lesssim\left\|\left(\phi_{t}, \psi_{t}, \zeta_{t}\right)(0)\right\|^{2}+\mathrm{e}^{-c \delta \beta}+(\delta+N(t)) \int_{0}^{t}\left(\|(\phi, \psi, \zeta)(\tau)\|_{2}^{2}+\left\|\omega_{t x x}(\tau)\right\|^{2}\right) d \tau
\end{aligned}
$$

where for the last inequality, we employed the following boundary estimate:

$$
\begin{aligned}
& \int_{0}^{t}\left(\psi_{t}^{2}+\omega_{t}^{2}+\left|\omega_{t x} \omega_{t}\right|\right)(0, \tau) d \tau \\
\lesssim & \int_{0}^{t} \delta \mathrm{e}^{-c \delta \beta} \mathrm{e}^{-c \delta \tau}\left(1+\left|\omega_{t x}\right|(0, \tau)\right) d \tau \\
\lesssim & \int_{0}^{t} \delta \mathrm{e}^{-c \delta \beta} \mathrm{e}^{-c \delta \tau}\left(1+\left\|\omega_{t x}\right\|^{\frac{1}{2}}\left\|\omega_{t x x}\right\|^{\frac{1}{2}}\right) d \tau \\
\lesssim & \mathrm{e}^{-c \delta \beta}+\delta \int_{0}^{t}\left\|\left(\omega_{t x}, \omega_{t x x}\right)(\tau)\right\|^{2} d \tau
\end{aligned}
$$

By Lemmas 2.1, 3.1 and 3.2, we can get (4.1) from (4.6). 
Based on the estimates on the time derivative, in order to derive the first order energy estimates, we need to control boundary integral terms with respect to the spatial derivatives first. Let us rewrite system (2.14) as follows:

$$
\left\{\begin{array}{l}
u \phi_{x}+\rho \psi_{x}=Q_{1}-\phi_{t} \\
u \psi_{x}+R \zeta_{x}+\frac{R \theta}{\rho} \phi_{x}=Q_{2}-\psi_{t} \\
C_{v} u \zeta_{x}+R \theta \psi_{x}=Q_{3}-C_{v} \zeta_{t}-w_{x}
\end{array}\right.
$$

So

$$
\begin{aligned}
\psi_{x} & =\frac{u\left(Q_{2}-\psi_{t}\right)}{u^{2}-R \gamma \theta}-\frac{R \theta\left(Q_{1}-\phi_{t}\right)}{\rho\left(u^{2}-R \gamma \theta\right)}-\frac{\gamma-1}{u^{2}-R \gamma \theta}\left(Q_{3}-C_{v} \zeta_{t}-w_{x}\right) \\
& =O(1)\left|\left(Q_{1}, Q_{2}, Q_{3}, \phi_{t}, \psi_{t}, \zeta_{t}, w_{x}\right)\right| .
\end{aligned}
$$

Therefore, based on the expressions in (2.15), estimate (3.27), Lemmas 2.1, 3.1, 3.2 and 4.1 , we obtain

$$
\begin{aligned}
& \int_{0}^{t} \psi_{x}^{2}(0, \tau) d \tau \lesssim \int_{0}^{t}\left|\left(Q_{1}, Q_{2}, Q_{3}, \phi_{t}, \psi_{t}, \zeta_{t}, w_{x}\right)\right|^{2}(0, \tau) d \tau \\
\lesssim & \left\|\left(\Phi_{0}, \Psi_{0}, W_{0}\right)\right\|^{2}+\left\|\left(\phi_{0}, \psi_{0}, \zeta_{0}\right)\right\|^{2}+\left\|\left(\phi_{t}, \psi_{t}, \zeta_{t}\right)(0)\right\|^{2}+\mathrm{e}^{-\delta \beta} \\
& +(\delta+N(t)) \int_{0}^{t}\left(\|(\phi, \psi, \zeta)(\tau)\|_{2}^{2}+\left\|\omega_{t x x}(\tau)\right\|^{2}\right) d \tau, \\
& \int_{0}^{t}\left|\left(\phi_{x}, \zeta_{x}\right)\right|^{2}(0, \tau) d \tau \\
\lesssim & \int_{0}^{t}\left|\left(Q_{1}, Q_{3}\right)\right|^{2}(0, \tau) d \tau+\int_{0}^{t}\left|\left(\phi_{t}, \zeta_{t}, w_{x}, \psi_{x}\right)\right|^{2}(0, \tau) d \tau \\
\lesssim & \left\|\left(\Phi_{0}, \Psi_{0}, W_{0}\right)\right\|^{2}+\left\|\left(\phi_{0}, \psi_{0}, \zeta_{0}\right)\right\|^{2}+\left\|\left(\phi_{t}, \psi_{t}, \zeta_{t}\right)(0)\right\|^{2}+\mathrm{e}^{-\delta \beta} \\
& +(\delta+N(t)) \int_{0}^{t}\left(\|(\phi, \psi, \zeta)(\tau)\|_{2}^{2}+\left\|\omega_{t x x}(\tau)\right\|^{2}\right) d \tau, \\
& \int_{0}^{t} \omega_{x x}^{2}(0, \tau) d \tau \lesssim \int_{0}^{t}\left[\omega^{2}+\zeta_{x}^{2}+\left|\theta_{x}^{s} \zeta\right|^{2}+\left(q_{x x}^{s}\right)^{2}\right](0, \tau) d \tau \\
\lesssim & \left\|\left(\Phi_{0}, \Psi_{0}, W_{0}\right)\right\|^{2}+\left\|\left(\phi_{0}, \psi_{0}, \zeta_{0}\right)\right\|^{2}+\left\|\left(\phi_{t}, \psi_{t}, \zeta_{t}\right)(0)\right\|^{2} \\
& +\mathrm{e}^{-\delta \beta}+(\delta+N(t)) \int_{0}^{t}\left(\|(\phi, \psi, \zeta)(\tau)\|_{2}^{2}+\left\|\omega_{t x x}(\tau)\right\|^{2}\right) d \tau .
\end{aligned}
$$

Now we are ready to establish the first-order energy estimate. 
Lemma 4.2. Under the assumption of Proposition 2.2, if $N(t)$ and $\delta$ are suitably small, it holds that

$$
\begin{aligned}
& \left\|\left(\phi_{x}, \psi_{x}, \zeta_{x}\right)(t)\right\|^{2}+\int_{0}^{t}\left(\left|\left(\phi_{x}, \psi_{x}, \zeta_{x}, \omega_{x x}\right)\right|^{2}(0, \tau)+\left\|w_{x}(\tau)\right\|_{1}^{2}\right) d \tau \\
\lesssim & \left\|\left(\Phi_{0}, \Psi_{0}, W_{0}\right)\right\|^{2}+\left\|\left(\phi_{0}, \psi_{0}, \zeta_{0}\right)\right\|_{1}^{2}+\left\|\left(\phi_{t}, \psi_{t}, \zeta_{t}\right)(0)\right\|^{2}+\mathrm{e}^{-\delta \beta} \\
+ & (\delta+N(t)) \int_{0}^{t}\left(\|(\phi, \psi, \zeta)(\tau)\|_{2}^{2}+\left\|\omega_{t x x}(\tau)\right\|^{2}\right) d \tau .
\end{aligned}
$$

Proof. Multiplying the equations in (2.14) by $-\frac{R \theta}{\rho} \phi_{x x},-\rho \psi_{x x},-\frac{\rho \tau_{x x}}{\theta}$ and $-\frac{\rho \omega_{x x}}{4 b \theta^{4}}$ one by one, respectively, we have

$$
\begin{aligned}
& \left(\frac{R \theta}{\rho} \frac{\phi_{x}^{2}}{2}\right)_{t}-\left(\frac{R \theta}{\rho} \phi_{x} \phi_{t}+\frac{R \theta u}{\rho} \frac{\phi_{x}^{2}}{2}\right)_{x} \\
= & \left\{\left(\frac{R \theta}{2 \rho}\right)_{t}-\left(\frac{R \theta u}{2 \rho}\right)_{x}\right\} \phi_{x}^{2}-\frac{R \theta}{\rho} \phi_{x x} Q_{1}-\left(\frac{R \theta}{\rho}\right)_{x} \phi_{t} \phi_{x} \\
& \left(\frac{\rho \psi_{x}^{2}}{2}\right)_{t}-\left(\rho u \frac{\psi_{x}^{2}}{2}+\rho \psi_{t} \psi_{x}\right)_{x}-R \theta \phi_{x} \psi_{x x}-R \rho \zeta_{x} \psi_{x x} \\
= & -\rho \psi_{x x} Q_{2}-(\rho u)_{x} \psi_{x}^{2}, \\
& \left(\frac{C_{v} \rho}{\theta} \frac{\zeta_{x}^{2}}{2}\right)_{t}-\left(\frac{C_{v} \rho u}{\theta} \frac{\zeta_{x}^{2}}{2}+\frac{C_{v} \rho}{\theta} \zeta_{t} \zeta_{x}\right)_{x}-R \rho \zeta_{x x} \psi_{x}-\frac{\rho \zeta_{x x}}{\theta} \omega_{x} \\
= & \left\{\left(\frac{C_{v} \rho}{\theta}\right)_{t}-\left(\frac{C_{v} \rho u}{\theta}\right)_{x}\right\} \frac{\zeta_{x}^{2}}{2}-\frac{\rho \zeta_{x x}}{\theta} Q_{3}-\left(\frac{C_{v} \rho}{\theta}\right)_{x} \zeta_{t} \zeta_{x}, \\
& \frac{\rho \omega_{x x}^{2}}{4 b \theta^{4}}-\left(\frac{a \rho \omega \omega_{x}}{4 b \theta^{4}}\right)_{x}+\frac{a \rho \omega_{x}^{2}}{4 b \theta^{4}}+\left(\frac{a \rho}{4 b \theta^{4}}\right)_{x} \omega \omega_{x}-\frac{\rho \zeta_{x}}{\theta} \omega_{x x} \\
= & \left\{4 b \theta_{x}^{s} \zeta\left[\theta^{2}+\theta \theta^{s}+\left(\theta^{s}\right)^{2}\right]-q_{x x}^{s}\right\} \frac{\rho \omega_{x x}}{4 b \theta^{4}} .
\end{aligned}
$$

Adding them together, we get

$$
\left(\frac{R \theta}{\rho} \frac{\phi_{x}^{2}}{2}+\frac{\rho \psi_{x}^{2}}{2}+\frac{C_{v} \rho}{\theta} \frac{\zeta_{x}^{2}}{2}\right)_{t}+\frac{\rho \omega_{x x}^{2}}{4 b \theta^{4}}+\frac{a \rho \omega_{x}^{2}}{4 b \theta^{4}}+\left(\frac{a \rho}{4 b \theta^{4}}\right)_{x} \omega \omega_{x}-I_{6 x}=I_{7},
$$

where

$$
I_{6}:=\frac{R \theta}{\rho} \phi_{t} \phi_{x}+\frac{R \theta u}{2 \rho} \phi_{x}^{2}+\rho u \frac{\psi_{x}^{2}}{2}+\rho \psi_{t} \psi_{x}+R \theta \phi_{x} \psi_{x}
$$




$$
\begin{aligned}
&+R \rho \zeta_{x} \psi_{x}+\frac{C_{v} \rho}{\theta} \zeta_{t} \zeta_{x}+\frac{C_{v} \rho u}{\theta} \frac{\zeta_{x}^{2}}{2}-\frac{a \rho \omega \omega_{x}}{4 b \theta^{4}}-\frac{\rho \zeta_{x}}{\theta} \omega_{x} \\
& \lesssim O(1)\left|\left(\phi_{x}, \psi, \zeta_{x}, \phi_{t}, \psi_{t}, \zeta_{t}, \omega, \omega_{x}\right)\right|^{2}, \\
& I_{7}:=\left\{\left(\frac{R \theta}{2 \rho}\right)_{t}-\left(\frac{R \theta u}{2 \rho}\right)_{x}\right\} \phi_{x}^{2}-R \theta_{x} \psi_{x} \phi_{x}-R \rho_{x} \zeta_{x} \psi_{x}-(\rho u)_{x} \psi_{x}^{2} \\
&+\left\{\left(\frac{C_{v} \rho}{\theta}\right)_{t}-\left(\frac{C_{v} \rho u}{\theta}\right)_{x}\right\} \frac{\zeta_{x}^{2}}{2}-\frac{R \theta}{\rho} \phi_{x x} Q_{1}-\left(\frac{R \theta}{\rho}\right)_{x} \phi_{t} \phi_{x} \\
& \quad-\rho \psi_{x x} Q_{2}-\rho_{x} \psi_{t} \psi_{x}-\frac{\rho \zeta_{x x}}{\theta} Q_{3}-\left(\frac{C_{v} \rho}{\theta}\right)_{x} \zeta_{t} \zeta_{x}-\left(\frac{\rho}{\theta}\right)_{x} \zeta_{x} \omega_{x} \\
&+\left\{4 b \theta_{x}^{s} \zeta\left[\theta^{2}+\theta \theta^{s}+\left(\theta^{s}\right)^{2}\right]-q_{x x}^{s}\right\} \frac{\rho \omega_{x x}}{4 b \theta^{4}} \\
& \lesssim(\delta+N(t))\left|\left(\phi_{x x}, \psi_{x x}, \zeta_{x x}, \omega_{x x}, \phi_{x}, \psi_{x}, \zeta_{x}, \omega_{x}, \phi, \psi, \zeta, \omega\right)\right|^{2} .
\end{aligned}
$$

Therefore, integrating (4.18) over $\mathbb{R}_{+} \times[0, t]$ and using (4.10)-(4.12), we can get (4.13).

\subsection{Second-order energy estimates}

Now, we are going to derive the second-order energy estimates. First for the time derivatives, differentiating (2.14) with respect to $t$, we get the system that

$$
\left\{\begin{array}{l}
\phi_{t t}+u \phi_{t x}+\rho \psi_{t x}=\widetilde{Q}_{1}, \\
\psi_{t t}+u \psi_{t x}+R \zeta_{t x}+\frac{R \theta}{\rho} \phi_{t x}=\widetilde{Q}_{2} \\
C_{v} \zeta_{t t}+C_{v} u \zeta_{t x}+R \theta \psi_{t x}+\omega_{t x}=\widetilde{Q}_{3} \\
-\omega_{t x x}+a \omega_{t}+4 b \theta^{3} \zeta_{t x}+4 b \theta_{x}^{s} \zeta_{t}\left(\theta^{2}+\theta \theta^{s}+\left(\theta^{s}\right)^{2}\right)=\widetilde{Q}_{4}
\end{array}\right.
$$

where

$$
\begin{aligned}
& \widetilde{Q}_{1}=Q_{1 t}-\left(u_{t} \phi_{x}+\rho_{t} \psi_{x}\right), \\
& \widetilde{Q}_{2}=Q_{2 t}-u_{t} \psi_{x}-\left(\frac{R \theta}{\rho}\right)_{t} \phi_{x}, \\
& \widetilde{Q}_{3}=Q_{3 t}-C_{v} u_{t} \zeta_{x}-R \theta_{t} \psi_{x}, \\
& \widetilde{Q}_{4}=q_{x x}^{s}-4 b\left(\theta^{3}\right)_{t} \zeta_{x}-4 b\left\{\theta_{x}^{s}\left[\theta^{2}+\theta \theta^{s}+\left(\theta^{s}\right)^{2}\right]\right\}_{t} \zeta .
\end{aligned}
$$

Then we have the following lemma on the estimates of the time derivatives. 
Lemma 4.3. Under the assumptions of Proposition 2.2, if $N(t)$ and $\delta$ are suitably small, it holds that

$$
\begin{aligned}
& \|\left(\phi_{t t}, \psi_{t t}, \zeta_{t t}\right)(t) \|^{2}+\int_{0}^{t}\left[\left(\phi_{t t}^{2}+\zeta_{t t}^{2}\right)(0, \tau)+\left\|\left(\omega_{t t}, \omega_{t t x}\right)(\tau)\right\|^{2}\right] d \tau \\
& \lesssim\left\|\left(\Phi_{0}, \Psi_{0}, W_{0}\right)\right\|^{2}+\left\|\left(\phi_{0}, \psi_{0}, \zeta_{0}\right)\right\|_{2}^{2}+\left\|\left(\phi_{t}, \psi_{t}, \zeta_{t}\right)(0)\right\|_{1}^{2} \\
& \quad+\left\|\left(\phi_{t t}, \psi_{t t}, \zeta_{t t}\right)(0)\right\|^{2}+\mathrm{e}^{-\delta \beta}+\delta\left\|\left(\omega_{t x}, \omega_{t x x}\right)(t)\right\|^{2} \\
& \quad+(\delta+N(t)) \int_{0}^{t}\left(\|(\phi, \psi, \zeta)(\tau)\|_{2}^{2}+\left\|\omega_{t x}(\tau)\right\|_{1}^{2}\right) d \tau .
\end{aligned}
$$

Proof. Differentiating the equations in (4.19) with respect to $t$, then multiplying the resulted equations one by one by $\frac{R \theta}{\rho} \phi_{t t}, \rho \psi_{t t}, \frac{\rho}{\theta} \zeta_{t t}$ and $\frac{\rho}{4 b \theta^{4}} w_{t t}$ respectively, and adding them together, we get

$$
\left(\frac{R \theta}{2 \rho} \phi_{t t}^{2}+\frac{\rho}{2} \psi_{t t}^{2}+\frac{C_{v} \rho}{2 \theta} \zeta_{t t}^{2}\right)_{t}+\frac{\rho w_{x t t}^{2}}{4 b \theta^{4}}+\left(\frac{\rho}{4 b \theta^{4}}\right)_{x} w_{t t} w_{x t t}+\frac{a \rho w_{t t}^{2}}{4 b \theta^{4}}+J_{1 x}=J_{2},
$$

where

$$
\begin{aligned}
J_{1}:= & \frac{R \theta u}{2 \rho} \phi_{t t}^{2}+\frac{\rho u}{2} \psi_{t t}^{2}+\frac{C_{v} \rho u}{2 \theta} \zeta_{t t}^{2}+R \theta \phi_{t t} \psi_{t t}+R \rho \psi_{t t} \zeta_{t t} \\
& +\frac{\rho}{\theta} \zeta_{t t} w_{t t}-\frac{\rho}{4 b \theta^{4}} w_{t t} w_{x t t} \\
J_{2}:=\{ & \left.\left(\frac{R \theta}{2 \rho}\right)_{t}+\left(\frac{R \theta u}{2 \rho}\right)_{x}\right\} \phi_{t t}^{2}+\left(\widetilde{Q}_{1 t}-\rho_{t} \psi_{t x}-u_{t} \phi_{t x}\right) \frac{R \theta}{\rho} \phi_{t t} \\
& +\left[\widetilde{Q}_{2 t}-u_{t} \psi_{t x}-\left(\frac{R \theta}{\rho}\right)_{t} \phi_{t x}\right] \rho \psi_{t t}+R \theta_{x} \phi_{t t} \psi_{t t}-R \rho_{x} \psi_{t t} \zeta_{t t} \\
& +\left\{\left(\frac{C_{v} \rho}{2 \theta}\right)_{t}+\left(\frac{C_{v} \rho u}{2 \theta}\right)_{x}\right\} \zeta_{t t}^{2}+\left(Q_{3 t}-C_{v} u_{t} \zeta_{t x}-R \theta_{t} \psi_{t x}\right) \frac{\rho}{\theta} \zeta_{t t} \\
& +\left\{\widetilde{Q}_{4}-12 b \theta^{2} \theta_{t} \zeta_{t x}-\left[4 b \theta_{x}^{s} \zeta_{t}\left(\theta^{2}+\theta \theta^{s}+\left(\theta^{s}\right)^{2}\right)\right]_{t}\right\} \frac{w_{t t}}{4 b \theta^{4}}
\end{aligned}
$$

In particular, one has

$$
\begin{aligned}
& J_{1}(0, \tau) \geq c\left(\phi_{t t}^{2}+\zeta_{t t}^{2}\right)(0, \tau)-C\left(\psi_{t t}^{2}+w_{t t}^{2}\right)(0, \tau)-\frac{\rho_{-}}{4 b \theta_{-}^{4}} \int_{0}^{t} w_{t t} w_{x t t}(0, \tau) d \tau, \\
& \left|J_{2}\right| \lesssim O(1)(\delta+N(t))\left|\left(\phi_{t t}, \psi_{t t}, \zeta_{t t}, \phi_{t x}, \psi_{t x}, \zeta_{t x}, \phi_{x}, \psi_{x}, \zeta_{x}, \phi, \psi, \zeta\right)\right|^{2} .
\end{aligned}
$$

Integrating (4.22) over $\mathbb{R}_{+}$, we obtain

$$
\frac{d}{d t}\left\|\left(\phi_{t t}, \psi_{t t}, \zeta_{t t}\right)(t)\right\|^{2}+\left(\phi_{t t}^{2}+\zeta_{t t}^{2}\right)(0, t)+\left\|\left(w_{t t}, w_{t t x}\right)(t)\right\|^{2}
$$




$$
\begin{aligned}
& \lesssim\left(\psi_{t t}^{2}+w_{t t}^{2}\right)(0, t)+\frac{\rho_{-}}{4 b \theta_{-}^{4}} w_{t t} w_{x t t}(0, t) \\
& \quad+O(1)(\delta+N(t))\left\|\left(\phi_{t t}, \psi_{t t}, \zeta_{t t}, \phi_{t x}, \psi_{t x}, \zeta_{t x}, \phi_{x}, \psi_{x}, \zeta_{x}, \phi, \psi, \zeta\right)(t)\right\|^{2}
\end{aligned}
$$

Note that

$$
\begin{aligned}
& \int_{0}^{t} \frac{\rho}{4 b \theta^{4}} w_{t t} w_{x t t}(0, \tau) d \tau=\frac{\rho_{-}}{4 b \theta_{-}^{4}} \int_{0}^{t} w_{t t} w_{x t t}(0, \tau) d \tau \\
= & \frac{\rho_{-}}{4 b \theta_{-}^{4}} \int_{0}^{t}\left\{\left(w_{t t} w_{x t}\right)_{t}-w_{t t t} w_{x t}\right\}(0, \tau) d \tau \\
\lesssim & \left|w_{t t} w_{x t}\right|(0, t)+\left|w_{t t} w_{x t}\right|(0,0)-\frac{\rho_{-}}{4 b \theta_{-}^{4}} \int_{0}^{t} w_{t t t} w_{x t}(0, \tau) d \tau \\
\lesssim & \delta\left\|\left(w_{t x}, w_{t x x}\right)(t)\right\|^{2}+\delta \int_{0}^{t} w_{t x}^{2}(0, \tau) d \tau+\delta \\
\lesssim & \delta\left\|\left(w_{t x}, w_{t x x}\right)(t)\right\|^{2}+\delta \int_{0}^{t}\left\|\left(w_{t x}, w_{t x x}\right)(\tau)\right\|^{2} d \tau+\delta
\end{aligned}
$$

Integrating (4.27) on $[0, t]$ and using (4.28), we can get (4.21).

To control the derivatives with respect to $\partial_{t x}$ on the boundary, we rewrite system (4.19) as follows:

$$
\left\{\begin{array}{l}
u \phi_{t x}+\rho \psi_{t x}=\widetilde{Q}_{1}-\phi_{t t} \\
u \psi_{t x}+R \zeta_{t x}+\frac{R \theta}{\rho} \phi_{t x}=\widetilde{Q}_{2}-\psi_{t t} \\
C_{v} u \zeta_{t x}+R \theta \psi_{t x}=\widetilde{Q}_{3}-C_{v} \zeta_{t t}-w_{t x}
\end{array}\right.
$$

Then

$$
\begin{aligned}
& \int_{0}^{t} w_{t x}^{2}(0, \tau) d \tau \lesssim \int_{0}^{t}\left\|w_{t x}(\tau)\right\|\left\|w_{t x x}(\tau)\right\| d \tau \\
\lesssim & v \int_{0}^{t}\left\|w_{t x x}(\tau)\right\|^{2} d \tau+C_{v} \int_{0}^{t}\left\|w_{t x}(\tau)\right\|^{2} d \tau, \\
& \int_{0}^{t}\left|\left(\phi_{t x}, \psi_{t x}, \zeta_{t x}\right)\right|^{2}(0, \tau) d \tau \\
\lesssim & \int_{0}^{t}\left|\left(\phi_{t t}, \psi_{t t}, \zeta_{t t}, w_{t x}\right)\right|^{2}(0, \tau) d \tau+\int_{0}^{t}\left|\left(\widetilde{Q}_{1}, \widetilde{Q}_{2}, \widetilde{Q}_{3}\right)\right|^{2}(0, \tau) d \tau \\
\lesssim & \left\|\left(\Phi_{0}, \Psi_{0}, W_{0}\right)\right\|^{2}+\left\|\left(\phi_{0}, \psi_{0}, \zeta_{0}\right)\right\|_{2}^{2}+\left\|\left(\phi_{t}, \psi_{t}, \zeta_{t}\right)(0)\right\|_{1}^{2}+\mathrm{e}^{-\delta \beta} \\
& +(v+\delta+N(t)) \int_{0}^{t}\left(\|(\phi, \psi, \zeta)(\tau)\|_{2}^{2}+\left\|\omega_{t x x}(\tau)\right\|^{2}\right) d \tau,
\end{aligned}
$$


where $v>0$ is a constant small enough. Therefore, one also has

$$
\begin{aligned}
& \int_{0}^{t}\left|w_{t x x}\right|^{2}(0, \tau) d \tau \lesssim \int_{0}^{t}\left|\left(\omega_{t}, \zeta_{t x}, \theta_{x}^{s} \zeta_{t}, \zeta_{,} \zeta_{t}, \widetilde{Q}_{4}\right)\right|^{2}(0, \tau) d \tau \\
& \lesssim\left\|\left(\Phi_{0}, \Psi_{0}, W_{0}\right)\right\|^{2}+\left\|\left(\phi_{0}, \psi_{0}, \zeta_{0}\right)\right\|_{2}^{2}+\left\|\left(\phi_{t}, \psi_{t}, \zeta_{t}\right)(0)\right\|_{1}^{2} \\
& \quad+e^{-\delta \beta}+\left\|\left(\phi_{t t}, \psi_{t t}, \zeta_{t t}\right)(0)\right\|^{2} \\
& \quad+(v+\delta+N(t)) \int_{0}^{t}\left(\|(\phi, \psi, \zeta)(\tau)\|_{2}^{2}+\left\|\omega_{t x x}(\tau)\right\|^{2}\right) d \tau .
\end{aligned}
$$

Now we are ready to introduce the following lemma.

Lemma 4.4. Under the assumption of Proposition 2.2, if $N(t)$ and $\delta$ are suitably small, it holds that

$$
\begin{aligned}
& \left\|\left(\phi_{t x}, \psi_{t x}, \zeta_{t x}\right)(t)\right\|^{2}+\int_{0}^{t}\left(\left|\left(\phi_{t x}, \psi_{t x}, \zeta_{t x}, w_{t x}, w_{t x x}\right)\right|^{2}(0, \tau)+\left\|w_{t x}(\tau)\right\|_{1}^{2}\right) d \tau \\
& \lesssim\left\|\left(\Phi_{0}, \Psi_{0}, W_{0}\right)\right\|^{2}+\left\|\left(\phi_{0}, \psi_{0}, \zeta_{0}\right)\right\|_{2}^{2}+\left\|\left(\phi_{t}, \psi_{t}, \zeta_{t}\right)(0)\right\|_{1}^{2}+\mathrm{e}^{-\delta \beta} \\
& \quad+\left\|\left(\phi_{t t}, \psi_{t t}, \zeta_{t t}\right)(0)\right\|^{2}+(\delta+N(t)) \int_{0}^{t}\|(\phi, \psi, \zeta)(\tau)\|_{2}^{2} d \tau
\end{aligned}
$$

Proof. Differentiating the equations in (4.19) with respect to $x$, then multiplying each of the resulting equations by $\frac{R \theta}{\rho} \phi_{t x}, \rho \psi_{t x}, \frac{\rho}{\theta} \zeta_{t x}$ and $\frac{\rho}{4 b \theta^{4}} w_{t x}$ respectively, and adding all resulting equations together, we get

$$
\left(\frac{R \theta}{2 \rho} \phi_{t x}^{2}+\frac{\rho}{2} \psi_{t x}^{2}+\frac{C_{v} \rho}{2 \theta} \zeta_{t x}^{2}\right)_{t}+\frac{\rho w_{t x x}^{2}}{4 b \theta^{4}}+\left(\frac{\rho}{4 b \theta^{4}}\right)_{x} w_{t x} w_{t x x}+\frac{a \rho w_{t x}^{2}}{4 b \theta^{4}}+J_{3 x}=J_{4},
$$

where

$$
\begin{aligned}
J_{3}:= & \frac{R \theta u}{2 \rho} \phi_{t x}^{2}+\frac{\rho u}{2} \psi_{t x}^{2}+\frac{C_{v} \rho u}{2 \theta} \zeta_{t x}^{2}+R \theta \phi_{t x} \psi_{t x}+R \rho \psi_{t x} \zeta_{t x} \\
& +\frac{\rho}{\theta} \zeta_{t x} w_{t x}-\frac{\rho}{4 b \theta^{4}} w_{t x} w_{t x x} \\
= & O(1)\left|\left(\phi_{t x}, \psi_{t x}, \zeta_{t x}, w_{t x}, w_{t x x}\right)(x, t)\right|^{2} \\
J_{4}:=\{ & \left.\left(\frac{R \theta}{2 \rho}\right)_{t}+\left(\frac{R \theta u}{2 \rho}\right)_{x}\right\} \phi_{t x}^{2}+\left(\widetilde{Q}_{1 x}-\rho_{x} \psi_{t x}-u_{x} \phi_{t x}\right) \frac{R \theta}{\rho} \phi_{t x} \\
& +\left[\widetilde{Q}_{2 x}-u_{x} \psi_{t x}-\left(\frac{R \theta}{\rho}\right)_{x} \phi_{t x}\right] \rho \psi_{t x}+R \theta_{x} \phi_{t x} \psi_{t x}-R \rho_{x} \psi_{t x} \zeta_{t x} \\
& +\left\{\left(\frac{C_{v} \rho}{2 \theta}\right)_{t}+\left(\frac{C_{v} \rho u}{2 \theta}\right)_{x}\right\} \zeta_{t x}^{2}+\left(\widetilde{Q}_{3 x}-C_{v} u_{x} \zeta_{t x}-R \theta_{x} \psi_{t x}\right) \frac{\rho}{\theta} \zeta_{t x}
\end{aligned}
$$




$$
\begin{gathered}
+\left\{\widetilde{Q}_{4 x}-12 b \theta^{2} \theta_{x} \zeta_{t x}-\left[4 b \theta_{x}^{s} \zeta_{t}\left(\theta^{2}+\theta \theta^{s}+\left(\theta^{s}\right)^{2}\right)\right]_{x}\right\} \frac{w_{t x}}{4 b \theta^{4}} \\
=O(1)\left|\left(\phi_{t x}, \psi_{t x}, \zeta_{t x}, w_{t x}, \phi_{x x}, \psi_{x x}, \zeta_{x x}, w_{x x}, \phi_{x}, \psi_{x}, \zeta_{x}, w_{x}, \phi, \psi, \zeta, w\right)(x, t)\right|^{2} .
\end{gathered}
$$

Integrating (4.34) over $\mathbb{R}_{+} \times[0, t]$ and using the boundary estimates (4.30)-(4.32), we can get (4.33).

To control the other derivatives of second order, differentiating (3.11) with respect to $x$, we get the system that

$$
\left\{\begin{array}{l}
\phi_{t x}+u \phi_{x x}+\rho \psi_{x x}=Q_{5} \\
\psi_{t x}+u \psi_{x x}+R \zeta_{x x}+\frac{R \theta}{\rho} \phi_{x x}=Q_{6} \\
C_{v} \zeta_{t x}+C_{v} u \zeta_{x x}+R \theta \psi_{x x}+\omega_{x x}=Q_{7} \\
-\omega_{x x x}+a \omega_{x}+4 b \theta^{3} \zeta_{x x}+4 b \theta_{x}^{s} \zeta_{x}\left(\theta^{2}+\theta \theta^{s}+\left(\theta^{s}\right)^{2}\right)=Q_{8}
\end{array}\right.
$$

where

$$
\begin{aligned}
& Q_{5}=Q_{1 x}-\left(u_{x} \phi_{x}+\rho_{x} \psi_{x}\right), \\
& Q_{6}=Q_{2 x}-u_{x} \psi_{x}-\left(\frac{R \theta}{\rho}\right)_{x} \phi_{x} \\
& Q_{7}=Q_{3 t}-C_{v} u_{t} \zeta_{x}-R \theta_{t} \psi_{x}, \\
& Q_{8}=q_{x x x}^{s}-4 b\left(\theta^{3}\right)_{x} \zeta_{x}-4 b\left\{\theta_{x}^{s}\left[\theta^{2}+\theta \theta^{s}+\left(\theta^{s}\right)^{2}\right]\right\}_{x} \zeta .
\end{aligned}
$$

It can be rewritten as

$$
\left\{\begin{array}{l}
u \phi_{x x}+\rho \psi_{x x}=Q_{5}-\phi_{t x} \\
u \psi_{x x}+R \zeta_{x x}+\frac{R \theta}{\rho} \phi_{x x}=Q_{6}-\psi_{t x} \\
C_{v} u \zeta_{x x}+R \theta \psi_{x x}=Q_{7}-C_{v} \zeta_{t x}-w_{x x} .
\end{array}\right.
$$

Therefore,

$$
\begin{aligned}
& \int_{0}^{t}\left|\left(\phi_{x x}, \psi_{x x}, \zeta_{x x}\right)\right|^{2}(0, \tau) d \tau \\
\lesssim & \int_{0}^{t}\left|\left(\phi_{t x}, \psi_{t x}, \zeta_{t x}, w_{x x}\right)\right|^{2}(0, \tau) d \tau+\int_{0}^{t}\left|\left(Q_{5}, Q_{6}, Q_{7}\right)\right|^{2}(0, \tau) d \tau \\
\lesssim & \left\|\left(\Phi_{0}, \Psi_{0}, W_{0}\right)\right\|^{2}+\left\|\left(\phi_{0}, \psi_{0}, \zeta_{0}\right)\right\|_{2}^{2}+\left\|\left(\phi_{t}, \psi_{t}, \zeta_{t}\right)(0)\right\|_{1}^{2} \\
& \quad+\mathrm{e}^{-\delta \beta}+(\delta+N(t)) \int_{0}^{t}\|(\phi, \psi, \zeta)(\tau)\|_{2}^{2} d \tau,
\end{aligned}
$$




$$
\begin{aligned}
& \int_{0}^{t}\left|\omega_{x x x}\right|^{2}(0, \tau) d \tau \\
\lesssim & \int_{0}^{t}\left|\left(\omega_{x}, \zeta_{x x}, \theta_{x}^{s} \zeta_{x}, \zeta, \zeta_{x}, Q_{8}\right)\right|^{2}(0, \tau) d \tau \\
\lesssim & \left\|\left(\Phi_{0}, \Psi_{0}, W_{0}\right)\right\|^{2}+\left\|\left(\phi_{0}, \psi_{0}, \zeta_{0}\right)\right\|_{2}^{2}+\left\|\left(\phi_{t}, \psi_{t}, \zeta_{t}\right)(0)\right\|_{1}^{2} \\
& \quad+\mathrm{e}^{-\delta \beta}+(\delta+N(t)) \int_{0}^{t}\|(\phi, \psi, \zeta)(\tau)\|_{2}^{2} d \tau .
\end{aligned}
$$

Then we have the following estimates.

Lemma 4.5. Under the assumption of Proposition 2.2, if $N(t)$ and $\delta$ are suitably small, it holds that

$$
\begin{aligned}
& \left\|\left(\phi_{x x}, \psi_{x x}, \zeta_{x x}\right)(t)\right\|^{2}+\int_{0}^{t}\left(\left|\left(\phi_{x x}, \psi_{x x}, \zeta_{x x}, w_{x x x}\right)\right|^{2}(0, \tau)+\left\|w_{x x}(\tau)\right\|_{1}^{2}\right) d \tau \\
\lesssim & \left\|\left(\Phi_{0}, \Psi_{0}, W_{0}\right)\right\|^{2}+\left\|\left(\phi_{0}, \psi_{0}, \zeta_{0}\right)\right\|_{2}^{2}+\left\|\left(\phi_{t}, \psi_{t}, \zeta_{t}\right)(0)\right\|_{1}^{2}+\mathrm{e}^{-\delta \beta} \\
& +\left\|\left(\phi_{t t}, \psi_{t t}, \zeta_{t t}\right)(0)\right\|^{2}+(\delta+N(t)) \int_{0}^{t}\|(\phi, \psi, \zeta)(\tau)\|_{2}^{2} d \tau
\end{aligned}
$$

Proof. Differentiating the equations in (2.14) with respect to $x$ twice, then multiplying each of them by $\frac{R \theta}{\rho} \phi_{x x}, \rho \psi_{x x},-\frac{\rho}{\theta} \zeta_{x x x}$ and $-\frac{\rho}{4 b \theta^{4}} w_{x x x}$ respectively, and adding the resulting equations together, we get

$$
\begin{aligned}
& \left(\frac{R \theta}{\rho} \frac{\phi_{x x}^{2}}{2}+\rho \frac{\psi_{x x}^{2}}{2}+\frac{C_{v} \rho}{2 \theta} \zeta_{x x}^{2}\right)_{t}+\frac{a \rho \omega_{x x}^{2}}{4 b \theta^{4}}+\rho \frac{\omega_{x x x}^{2}}{4 b \theta^{4}}+\left(\frac{a \rho}{4 b \theta^{4}}\right)_{x} \omega_{x x} \omega_{x x x}+J_{9 x} \\
= & O(1)(\delta+N(t))\left|\left(\phi_{x x}, \psi_{x x}, \zeta_{x x}, \omega_{x x x}\right)\right|\left|\left(\phi_{x x}, \psi_{x x}, \zeta_{x x}, \zeta_{t x}, \phi_{x}, \psi_{x}, \zeta_{x}, \phi, \psi, \zeta\right)\right|,
\end{aligned}
$$

where

$$
\begin{aligned}
J_{9}:= & \frac{R \theta u}{\rho} \frac{\phi_{x x}^{2}}{2}+\rho u \frac{\psi_{x x}^{2}}{2}+R \theta \psi_{x x} \phi_{x x}-\frac{C_{v} \rho u}{2 \theta} \zeta_{x x}^{2} \\
& -\frac{C_{v} \rho}{\theta} \zeta_{t x} \zeta_{x x}-\frac{\rho}{\theta} \omega_{x x} \zeta_{x x}-\frac{a \rho w_{x} w_{x x}}{4 b \theta^{4}} \\
= & O(1)\left|\left(\phi_{x x}, \psi_{x x}, \zeta_{x x}, \zeta_{t x}, \omega_{x}, \omega_{x x}\right)\right|^{2} .
\end{aligned}
$$

Integrating (4.43) over $\mathbb{R}_{+} \times[0, t]$ and using (4.40) with (4.41), we can get (4.42). This completes the proof.

Combining Lemmas 3.1-3.2 and 4.1-4.5 together, we can get

$$
\|(\Phi, \Psi, W)(t)\|^{2}+\|(\phi, \psi, \zeta)(t)\|_{2}^{2}+\left\|\left(\phi_{t}, \psi_{t}, \zeta_{t}\right)(t)\right\|_{1}^{2}+\left\|\left(\phi_{t t}, \psi_{t t}, \zeta_{t t}\right)(t)\right\|^{2}
$$




$$
\begin{aligned}
&+\int_{0}^{t}|(\Phi, \Psi, W)|^{2}(0, \tau) d \tau+\int_{0}^{t} \int_{\mathbb{R}_{+}}\left(\left|u_{x}^{s}\right||(\Psi, W)|^{2}+\Gamma^{2}+\Gamma_{x}^{2}\right) d x d \tau \\
&+\int_{0}^{t}\left|\left(\phi, \phi_{x}, \psi_{x}, \zeta_{x}, w_{x}, \phi_{x x}, \psi_{x x}, \zeta_{x x}, w_{x x}, w_{x x x}, \phi_{t x}, \psi_{t x}, \zeta_{t x}, w_{t x}, w_{t x x}\right)\right|^{2}(0, \tau) d \tau \\
&+\int_{0}^{t}\left(\|w(\tau)\|_{3}^{2}+\left\|w_{t}(\tau)\right\|_{2}^{2}\right) d \tau \\
& \lesssim\left\|\left(\Phi_{0}, \Psi_{0}, W_{0}\right)\right\|^{2}+\left\|\left(\phi_{0}, \psi_{0}, \zeta_{0}\right)\right\|_{1}^{2}+\left\|\left(\phi_{t}, \psi_{t}, \zeta_{t}\right)(0)\right\|_{1}^{2}+\left\|\left(\phi_{t t}, \psi_{t t}, \zeta_{t t}\right)(0)\right\|^{2} \\
& \quad+\mathrm{e}^{-\delta \beta}+(\delta+N(t)) \int_{0}^{t}\|(\phi, \psi, \zeta)(\tau)\|_{2}^{2} d \tau
\end{aligned}
$$

Finally in this section, we will deal with the term $\int_{0}^{t}\|(\phi, \psi, \zeta)(\tau)\|_{2}^{2} d \tau$, which appears in the right hand side of estimate (4.45).

Lemma 4.6. Under the assumptions of Proposition 2.2, if $N(t)$ and $\delta$ are suitably small, it holds that

$$
\begin{aligned}
\int_{0}^{t}\|(\phi, \psi, \zeta)(\tau)\|_{2}^{2} d \tau \lesssim & \left\|\left(\Phi_{0}, \Psi_{0}, W_{0}\right)\right\|^{2}+\left\|\left(\phi_{0}, \psi_{0}, \zeta_{0}\right)\right\|_{2}^{2} \\
& +\left\|\left(\phi_{t}, \psi_{t}, \zeta_{t}\right)(0)\right\|_{1}^{2}+\left\|\left(\phi_{t t}, \psi_{t t}, \zeta_{t t}\right)(0)\right\|^{2}+\mathrm{e}^{-\delta \beta} .
\end{aligned}
$$

Proof. Multiplying the second and the third equations in (2.39) by $R \theta^{s} \Phi_{x}$ and $2 \Psi_{x}$, respectively, we get

$$
\begin{gathered}
\left(R \theta^{s} \Psi \Phi_{x}\right)_{t}-R \theta_{t}^{s} \Psi \Phi_{x}-\left(R \theta^{s} \Psi \Phi_{t}\right)_{x}+R \theta_{x}^{s} \Psi \Phi_{t} \\
-R \theta^{s} \Psi_{x}^{2}-R \theta^{s} u_{x}^{s} \Phi \Psi_{x}+\left(R \theta^{s} \Phi_{x}\right)^{2} \\
=\left(G_{1}-R W_{x}-(\gamma-1) u_{x}^{s} \Psi+\frac{R \theta^{s}}{\rho^{s}} \rho_{x}^{s} \Phi\right) R \theta^{s} \Phi_{x}, \\
2 C_{v}\left[\left(W \Psi_{x}\right)_{t}-\left(W \Psi_{t}\right)_{x}\right]+2 C_{v} W_{x}\left(\Psi_{t}+u^{s} \Psi_{x}\right)+2 R \theta^{s} \Psi_{x}^{2} \\
-2 \Psi_{x}\left(\frac{q_{x}^{s}}{\rho^{s}} \Phi+\frac{p_{x}^{s}}{\rho^{s}} \Psi-\Gamma_{x}\right)=2 G_{2} \Psi_{x} .
\end{gathered}
$$

Then

$$
\begin{gathered}
\left(R \theta^{s} \Psi \Phi_{x}+2 C_{v} W \Psi_{x}\right)_{t}-\left(R \theta^{s} \Psi \Phi_{t}+2 C_{v} W \Psi_{t}\right)_{x} \\
+R \theta^{s} \Psi_{x}^{2}+\left(R \theta^{s} \Phi_{x}\right)^{2}-R \theta_{t}^{s} \Psi \Phi_{x}+R \theta_{x}^{s} \Psi \Phi_{t} \\
\quad-R \theta^{s} u_{x}^{s} \Phi \Psi_{x}+2 C_{v} W_{x}\left(\Psi_{t}+u^{s} \Psi_{x}\right) \\
=\left[G_{1}-R W_{x}-(\gamma-1) u_{x}^{s} \Psi+\frac{R \theta^{s}}{\rho^{s}} \rho_{x}^{s} \Phi\right] R \theta^{s} \Phi_{x} \\
+2 \Psi_{x}\left(\frac{q_{x}^{s}}{\rho^{s}} \Phi+\frac{p_{x}^{s}}{\rho^{s}} \Psi-\Gamma_{x}\right)+2 G_{2} \Psi_{x} .
\end{gathered}
$$


Multiplying the fourth equation in (2.39) by $W_{x}$, we get

$$
\frac{4 b\left(\theta^{s}\right)^{3}}{\rho} W_{x}^{2} \lesssim \Gamma_{x x}^{2}+\Gamma^{2}+\left(\theta_{x}^{s} \Phi\right)^{2}+\left(u_{x}^{s} \Psi\right)^{2}+G_{3}^{2} .
$$

Hence, integrating (4.49) over $\mathbb{R}_{+} \times[0, t]$ and by Lemma 2.1 and estimate (4.50), we obtain that

$$
\begin{aligned}
& \int_{0}^{t} \int_{\mathbb{R}_{+}}\left(R \theta^{s} \Psi_{x}^{2}+\left(R \theta^{s} \Phi_{x}\right)^{2}+W_{x}^{2}\right) d x d \tau \\
& \lesssim\left\|\left(\Psi, \Phi_{x}, W, \Psi_{x}\right)(t)\right\|^{2}+\left\|\left(\Psi, \Phi_{x}, W, \Psi_{x}\right)(0)\right\|^{2}+\mathrm{e}^{-c \delta \beta} \\
& \quad+\int_{0}^{t}|(\Phi, \Psi, W)|^{2}(0, \tau) d \tau+\int_{0}^{t} \int_{\mathbb{R}_{+}}\left(\left|u_{x}^{s}\right||(\Psi, W)|^{2}+\Gamma_{x x}^{2}+\Gamma^{2}+\left(u_{x}^{s}\right)^{2}\right) d x d \tau \\
& \quad+\int_{0}^{t}\left|\left(\Phi_{x}, \Psi_{x}, W_{x}\right)\right|^{2}(0, \tau) d \tau+(\delta+N(t)) \int_{0}^{t}\left\|\left(\phi_{x}, \psi_{x}, \zeta_{x}\right)(\tau)\right\|^{2} d \tau
\end{aligned}
$$

Recalling the relation between $(\phi, \psi, \zeta)$ and $(\Phi, \Psi, W)$ in $(2.18)$, we know

$$
\phi^{2}=\Phi_{x}^{2}, \quad \psi^{2} \lesssim \Psi_{x}^{2}+\left(u_{x}^{s} \Phi\right)^{2}, \quad \zeta^{2} \lesssim W_{x}^{2}+\left(\theta_{x}^{s} \Phi\right)^{2}+\left(u_{x}^{s} \Psi\right)^{2}+\rho^{2} \psi^{4}
$$

Therefore

$$
\begin{aligned}
& \int_{0}^{t}\|(\phi, \psi, \zeta)(\tau)\|^{2} d \tau \\
\lesssim & \left\|\left(\Phi_{0}, \Psi_{0}, W_{0}\right)\right\|^{2}+\left\|\left(\phi_{0}, \psi_{0}, \zeta_{0}\right)\right\|_{2}^{2}+\left\|\left(\phi_{t}, \psi_{t}, \zeta_{t}\right)(0)\right\|_{1}^{2} \\
& \quad+\left\|\left(\phi_{t t}, \psi_{t t}, \zeta_{t t}\right)(0)\right\|^{2}+\mathrm{e}^{-\delta \beta}+(\delta+N(t)) \int_{0}^{t}\left\|\left(\phi_{x}, \psi_{x}, \zeta_{x}\right)(\tau)\right\|_{1}^{2} d \tau .
\end{aligned}
$$

Furthermore, it follows from $(3.11)_{4}$ that

$$
\left|\zeta_{x}(x, t)\right|^{2} \lesssim\left|\left(\omega_{x}, \omega, \theta_{x}^{s} \zeta_{,} q_{x x}\right)(x, t)\right|^{2}
$$

In addition, multiplying $(3.11)_{2}$ and $(3.11)_{3}$ by $\frac{R \theta}{\rho} \phi_{x}$ and $2 \psi_{x}$, respectively, we obtain

$$
\begin{aligned}
& \left(\frac{R \theta}{\rho} \psi \phi_{x}+2 C_{v} \zeta \psi_{x}\right)_{t}-\left(\frac{R \theta}{\rho} \psi \phi_{t}+2 C_{v} \zeta \psi_{t}\right)_{x}+\left(\frac{R \theta}{\rho} \phi_{x}\right)^{2}+R \theta \psi_{x}^{2} \\
= & \left(\frac{R \theta}{\rho}\right)_{t} \psi \phi_{x}+\left(\frac{R \theta}{\rho}\right)_{x} \psi\left(u \phi_{x}+\rho \psi_{x}-Q_{1}\right)+C_{v} R \zeta_{x}^{2} \\
& +O(1)\left(\left|\zeta_{x} \phi_{x}\right|+\left|\psi_{x} \omega_{x}\right|+\left|\left(\phi_{x}, \zeta_{x}\right) Q_{2}\right|+\left|\psi_{x}\left(Q_{1}, Q_{3}\right)\right|\right) .
\end{aligned}
$$


Integrating (4.54) over $\mathbb{R}_{+} \times[0, t]$ and using (4.45) and (4.52) with (4.53), we have

$$
\begin{aligned}
& \int_{0}^{t} \int_{\mathbb{R}_{+}}\left(\psi_{x}^{2}+\psi_{x}^{2}+\zeta_{x}^{2}\right) d x d \tau \\
\lesssim & \left\|\left(\Phi_{0}, \Psi_{0}, W_{0}\right)\right\|^{2}+\left\|\left(\phi_{0}, \psi_{0}, \zeta_{0}\right)\right\|_{2}^{2}+\left\|\left(\phi_{t}, \psi_{t}, \zeta_{t}\right)(0)\right\|_{1}^{2} \\
& \quad+\left\|\left(\phi_{t t}, \psi_{t t}, \zeta_{t t}\right)(0)\right\|^{2}+\mathrm{e}^{-\delta \beta}+(\delta+N(t)) \int_{0}^{t}\|(\phi, \psi, \zeta)(\tau)\|_{2}^{2} d \tau .
\end{aligned}
$$

Next, from (4.37d), it holds that

$$
\left|\zeta_{x x}(x, t)\right|^{2} \lesssim\left|\left(\omega_{x x x}, \omega_{x}, \theta_{x}^{s} \zeta_{x}, Q_{8}\right)(x, t)\right|^{2}
$$

Finally, multiplying (4.37b) and (4.37c) by $\frac{R \theta}{\rho} \phi_{x x}$ and $2 \psi_{x x}$, respectively, we obtain

$$
\begin{aligned}
& \int_{0}^{t} \int_{\mathbb{R}_{+}}\left(\psi_{x x}^{2}+\psi_{x x}^{2}+\zeta_{x x}^{2}\right) d x d \tau \\
\lesssim & \left\|\left(\Phi_{0}, \Psi_{0}, W_{0}\right)\right\|^{2}+\left\|\left(\phi_{0}, \psi_{0}, \zeta_{0}\right)\right\|_{2}^{2}+\left\|\left(\phi_{t}, \psi_{t}, \zeta_{t}\right)(0)\right\|_{1}^{2} \\
& \quad+\left\|\left(\phi_{t t}, \psi_{t t}, \zeta_{t t}\right)(0)\right\|^{2}+\mathrm{e}^{-\delta \beta}+(\delta+N(t)) \int_{0}^{t}\|(\phi, \psi, \zeta)(\tau)\|_{2}^{2} d \tau .
\end{aligned}
$$

Combining the estimates (4.52), (4.55) and (4.57) together, we can get (4.46).

\section{Estimates on radiative perturbation}

In this section, we focus on the proof of the estimates related to the rediative heat flux.

Lemma 5.1. Under the assumptions in Proposition 2.2, if $N(t)$ and $\delta$ are suitably small, it holds that

$$
\begin{aligned}
& \|\Gamma(t)\|^{2}+\|w(t)\|_{3}^{2} \\
\lesssim & \left\|\left(\Phi_{0}, \Psi_{0}, W_{0}\right)\right\|^{2}+\left\|\left(\phi_{0}, \psi_{0}, \zeta_{0}\right)\right\|_{2}^{2} \\
& +\left\|\left(\phi_{t}, \psi_{t}, \zeta_{t}\right)(0)\right\|_{1}^{2}+\left\|\left(\phi_{t t}, \psi_{t t}, \zeta_{t t}\right)(0)\right\|^{2}+\mathrm{e}^{-\delta \beta} .
\end{aligned}
$$

Proof. By the last equation of (2.39), we see that

$$
-\Gamma_{x x}+a \Gamma+b \zeta\left(\theta+\theta^{s}\right)\left[\theta^{2}+\theta \theta^{s}+\left(\theta^{s}\right)^{2}\right]=q^{s} .
$$

Multiplying Eq. (5.2) by $\Gamma$, we have

$$
-\left(\Gamma_{x} \Gamma\right)_{x}+\Gamma_{x}^{2}+a \Gamma^{2}+b \Gamma \zeta\left(\theta+\theta^{s}\right)\left[\theta^{2}+\theta \theta^{s}+\left(\theta^{s}\right)^{2}\right]=q_{x}^{s} \Gamma .
$$


Integrating (5.2) over $[0,+\infty)$, and choosing $\delta$ and $N(t)$ suitably small, we have

$$
\left\|\left(\Gamma, \Gamma_{x}\right)(t)\right\|^{2} \lesssim \int_{0}^{\infty}\left[\zeta^{2}+\left(q_{x}^{s}\right)^{2}\right] d x+\left|\Gamma_{x} \Gamma(0, t)\right|
$$

Multiplying $(2.14)_{4}$ by $-w_{x x}$, we have

$$
\begin{aligned}
& -\left(a w w_{x}\right)_{x}+w_{x x}^{2}+a w_{x}^{2} \\
= & \left\{4 b \theta^{3} \zeta_{x}+4 b \theta_{x}^{s} \zeta-\left[\theta^{2}+\theta \theta^{s}+\left(\theta^{s}\right)^{2}\right]-q_{x x}^{s}\right\} w_{x x} .
\end{aligned}
$$

Integrating (5.5) over $[0,+\infty)$, and choosing $\delta$ and $N(t)$ suitably small, we have

$$
\left\|\left(w_{x}, w_{x x}\right)(t)\right\|^{2} \lesssim \int_{0}^{\infty}\left[\zeta_{x}^{2}+\zeta^{2}+\left(q_{x x}^{s}\right)^{2}\right] d x+\left|a w_{x} w(0, t)\right|
$$

Multiplying $(2.14 \mathrm{~d})$ by $-w_{x x x}$, we have

$$
\begin{aligned}
& -\left(a w_{x} w_{x x}\right)_{x}+w_{x x x}^{2}+a w_{x x}^{2} \\
= & \left\{4 b \theta^{3} \zeta_{x}+4 b \theta_{x}^{s} \zeta\left[\theta^{2}+\theta \theta^{s}+\left(\theta^{s}\right)^{2}\right]-q_{x x}^{s}\right\}_{x} w_{x x x} .
\end{aligned}
$$

Integrating (5.7) over $[0,+\infty)$, and choosing $\delta$ and $N(t)$ suitably small, we have

$$
\left\|\left(w_{x x}, w_{x x x}\right)(t)\right\|^{2} \lesssim \int_{0}^{\infty}\left[\zeta_{x x}^{2}+\zeta_{x}^{2}+\zeta^{2}+\left(q_{x x x}^{s}\right)^{2}\right] d x+\left|w_{x x} w_{x}(0, t)\right|
$$

Combining the estimates (5.4), (5.6) and (5.8) together, one has

$$
\begin{aligned}
& \|\Gamma(t)\|^{2}+\|w(t)\|_{3}^{2} \\
\lesssim & \|\zeta(t)\|_{2}^{2}+\int_{0}^{\infty}\left[\left(q_{x}^{s}\right)^{2}+\left(q_{x x}^{s}\right)^{2}+\left(q_{x x x}^{s}\right)^{2}\right] d x \\
& +\left|\Gamma_{x} \Gamma(0, t)\right|+\left|a w_{x} w(0, t)\right|+\left|a w_{x x} w_{x}(0, t)\right| .
\end{aligned}
$$

The last three terms on the right-hand side of (5.9) are estimated as follows one by one:

$$
\begin{aligned}
\left|\Gamma_{x} \Gamma(0, t)\right| & =|w(0, t)||\Gamma(0, t)| \lesssim\left|q^{s}(0, t)\right|\|\Gamma(t)\|_{\infty} \\
& \lesssim \delta\|\Gamma(t)\|^{\frac{1}{2}}\left\|\Gamma_{x}(t)\right\|^{\frac{1}{2}} \lesssim \delta\|\Gamma(t)\|^{2}+\delta\left\|\Gamma_{x}(t)\right\|^{\frac{2}{3}} \\
& \lesssim \delta\left\|\left(\Gamma, \Gamma_{x}\right)(t)\right\|^{2}+\delta \\
\left|w_{x} w\right|(0, t) & \lesssim\left|q^{S}(0, t)\right|\left\|w_{x}(t)\right\|_{\infty} \\
& \lesssim \delta\left\|w_{x}(t)\right\|^{\frac{1}{2}}\left\|w_{x x}(t)\right\|^{\frac{1}{2}} \lesssim \delta\left\|w_{x}(t)\right\|^{2}+\delta\left\|w_{x x}(t)\right\|^{\frac{2}{3}} \\
& \lesssim \delta\left\|\left(w_{x}, w_{x x}\right)(t)\right\|^{2}+\delta,
\end{aligned}
$$




$$
\begin{aligned}
&\left|w_{x x} w_{x}(0, t)\right|=\left|w_{x}\left\{a w+4 b \theta^{3} \zeta_{x}+4 b \theta_{x}^{s} \zeta\left[\theta^{2}+\theta \theta^{s}+\left(\theta^{s}\right)^{2}\right]-q_{x x}^{s}\right\}\right|(0, t) \\
& \lesssim\left|w_{x}\left(q_{x}^{s}, q_{x x}^{s}\right)\right|(0, t)+\left|w_{x}\left(\zeta_{x}, \zeta\right)\right|(0, t) \\
& \lesssim \delta\left\|\left(w_{x}, w_{x x}\right)(t)\right\|^{2}+\delta+\left\|w_{x}(t)\right\|_{\infty}\left\|\left(\zeta_{x}, \zeta\right)(t)\right\|_{\infty} \\
& \lesssim \delta\left\|\left(w_{x}, w_{x x}\right)(t)\right\|^{2}+\delta+\left\|w_{x}(t)\right\|^{\frac{1}{2}}\left\|w_{x x}(t)\right\|^{\frac{1}{2}} \\
& \\
& \quad \times\left(\left\|\left(\zeta_{x}, \zeta\right)(t)\right\|^{\frac{1}{2}}+\left\|\left(\zeta_{x}, \zeta_{x x}\right)(t)\right\|^{\frac{1}{2}}\right) \\
& \lesssim\left(\delta+\frac{1}{4}\right)\left\|\left(w_{x}, w_{x x}\right)(t)\right\|^{2}+\delta+\|\zeta(t)\|_{2}^{2}
\end{aligned}
$$

Inserting (5.10)-(5.12) into (5.9), we can get (5.1). This completes the proof of Lemma 5.1.

Lemma 5.2. Under the assumptions of Proposition 2.2, if $N(t)$ and $\delta$ are suitably small, it holds that

$$
\begin{aligned}
& \left\|w_{t}(t)\right\|_{2}^{2}+\left\|w_{t t}(t)\right\|_{1}^{2} \\
& \lesssim\left\|\left(\Phi_{0}, \Psi_{0}, W_{0}\right)\right\|^{2}+\left\|\left(\phi_{0}, \psi_{0}, \zeta_{0}\right)\right\|_{2}^{2} \\
& \quad+\left\|\left(\phi_{t}, \psi_{t}, \zeta_{t}\right)(0)\right\|_{1}^{2}+\left\|\left(\phi_{t t}, \psi_{t t}, \zeta_{t t}\right)(0)\right\|^{2}+\mathrm{e}^{-\delta \beta} .
\end{aligned}
$$

Proof. Multiplying (4.19d) by $w_{t}$, we get

$$
\begin{aligned}
& -\left(w_{t x} w_{t}\right)_{x}+w_{t x}^{2}+a w_{t}^{2} \\
= & -\left\{4 b \theta^{3} \zeta_{t x}+4 b \theta_{x}^{s} \zeta_{t}\left[\theta^{2}+\theta \theta^{s}+\left(\theta^{s}\right)^{2}\right]-\widetilde{Q}_{4}\right\} w_{t} .
\end{aligned}
$$

Multiplying (4.19d) by $-w_{t x x}$, we get

$$
\begin{aligned}
& -\left(a w_{t x} w_{t}\right)_{x}+w_{t x x}^{2}+a w_{t x}^{2} \\
= & \left\{4 b \theta^{3} \zeta_{t x}+4 b \theta_{x}^{s} \zeta_{t}\left[\theta^{2}+\theta \theta^{s}+\left(\theta^{s}\right)^{2}\right]-\widetilde{Q}_{4}\right\} w_{t x x} .
\end{aligned}
$$

Integrating (5.14) and (5.15) over $[0,+\infty)$, and choosing $\delta$ and $N(t)$ suitably small, it holds

$$
\left\|w_{t}(t)\right\|_{2}^{2} \lesssim \int_{0}^{\infty}\left(\zeta_{t x}^{2}+\zeta_{t}^{2}+\zeta^{2}+\widetilde{Q}_{4}^{2}\right) d x+\left|w_{t x} w_{t}(0, t)\right|
$$

where the last terms on the right-hand side of (5.16) are estimated as follows:

$$
\begin{aligned}
& \left|w_{t x} w_{t}(0, t)\right| \lesssim\left|q_{t}^{s}(0, t)\right|\left\|w_{t x}\right\|_{\infty} \\
\lesssim & \left|q_{t}^{s}(0, t)\right|\left\|w_{t x}\right\|^{\frac{1}{2}}\left\|w_{t x x}\right\|^{\frac{1}{2}} \lesssim \delta\left(\left\|w_{t x x}\right\|^{2}+\left\|w_{t x}\right\|^{2}\right)+\delta
\end{aligned}
$$


Plugging (5.17) into (5.16), we get

$$
\begin{aligned}
\left\|w_{t}(t)\right\|_{2}^{2} \lesssim & \left\|\left(\Phi_{0}, \Psi_{0}, W_{0}\right)\right\|^{2}+\left\|\left(\phi_{0}, \psi_{0}, \zeta_{0}\right)\right\|_{2}^{2}+\left\|\left(\phi_{t}, \psi_{t}, \zeta_{t}\right)(0)\right\|_{1}^{2} \\
& +\left\|\left(\phi_{t t}, \psi_{t t}, \zeta_{t t}\right)(0)\right\|^{2}+\mathrm{e}^{-\delta \beta}
\end{aligned}
$$

On the other hand, multiplying $(4.19 \mathrm{~d})$ by $-w_{t t}$, we get

$$
\begin{aligned}
& -\left(a w_{t t x} w_{t t}\right)_{x}+w_{t t x}^{2}+a w_{t t}^{2} \\
& =12 b \theta^{2} \theta_{t} \zeta_{t x} w_{t t}+\left(4 b \theta^{3} \zeta_{t t} w_{t t}\right)_{x}-12 b \theta^{2} \theta_{x} \zeta_{t t} w_{t t}-4 b \theta^{3} \tau_{t t} w_{t t x} \\
& \quad+\left\{4 b \theta_{x}^{s} \zeta_{t}\left[\theta^{2}+\theta \theta^{s}+\left(\theta^{s}\right)^{2}\right]-\widetilde{Q}_{4}\right\}_{t} w_{t t} .
\end{aligned}
$$

Integrating (5.19) over $[0,+\infty)$, and choosing $\delta$ and $N(t)$ suitably small, it holds

$$
\begin{aligned}
\left\|w_{t t}(t)\right\|_{1}^{2} & \lesssim\|(\zeta, \phi, \psi)(t)\|_{2}^{2}+\left|w_{t t} \zeta_{t t}(0, t)\right| \\
& \lesssim\|(\zeta, \phi, \psi)(t)\|_{2}^{2}+\delta .
\end{aligned}
$$

Combining (5.16) and (5.18) together, we can get (5.13). This completes the proof of Lemma 5.2 .

Now we can prove Proposition 2.2 to conclude the proof of Theorem 2.1 by Propositions 2.1 and 2.2.

Proof of Proposition 2.2. Using the results (4.45), (4.46), (5.1), and (5.13), we can obtain (2.48). This completes the proof of Proposition 2.2.

\section{Acknowledgements}

The research of Lili Fan was supported by the Natural Science Foundation of China (Grant 11871388) and in part by the Natural Science Foundation of China (Grant 11701439). The research of Lizhi Ruan was supported in part by the Natural Science Foundation of China (Grants 12171186, 11771169, 11871236, 11331005). The research of Wei Xiang was supported in part by the Research Grants Council of the HKSAR, China (Projects No. CityU 11303518, CityU 11304820, CityU 11300021). 


\section{References}

[1] C. Buet and B. Despres, Asymptotic analysis of fluid models for the coupling of radiation and hydrodynamics, J. Quant. Spectrosc. Radiat. Transfer 85 (2004), 385-418.

[2] J. Coulombel, T. Goudon, P. Lafitte and C. Lin, Analysis of large amplitude shock profiles for non-equilibrium radiative hydrodynamics: formation of Zeldovich spikes, Shock Waves 22 (2012), 181-197.

[3] R. Duan, L. Ruan and C. Zhu, Optimal decay rates to conservation laws with diffusiontype terms of regularity-gain and regularity-loss, Math. Models Methods Appl. Sci. 22 (2012), 1250012, 39 pp.

[4] L. Fan, L. Ruan and W. Xiang, Asymptotic stability of a composite wave of two viscous shock waves for the one-dimensional radiative Euler equations, Ann.I.H. Poincare-AN 36 (2019), 1-25.

[5] L. Fan, L. Ruan and W. Xiang, Asymptotic stability of rarefaction wave for the inflow problem governed by the one-dimensional radiative Euler equations, SIAM J. Math. Anal. 51 (2019), 595-625.

[6] L. Fan, L. Ruan and W. Xiang, Global stability of rarefaction wave for the outflow problem governed by the radiative Euler equations, Indiana Univ. Math. J. (2020), https: //www . iumj . indiana.edu/IUMJ/Preprints/8925.pdf.

[7] L. Fan, L. Ruan and W. Xiang, Asymptotic stability of viscous contact wave for the inflow problem of the one-dimensional radiative Euler equations, Discrete Contin. Dyn. Syst. Ser. A 41 (2021), 1971-1999.

[8] W. Gao, L. Ruan and C. Zhu, Decay rates to the planar rarefaction waves for a model system of the radiating gas in n-dimensions, J. Differential Equations 244 (2008), 26142640.

[9] P. Godillon-Lafitte and T. Goudon, A coupled model for radiative transfer: Doppler effects, equilibrium and non equilibrium diffusion asymptotics, SIAM J. Multiscale Model. Simul. 4 (2005), 1245-1279.

[10] K. Hamer, Nonlinear effects on the propagation of sound waves in a radiating gas, Quart. J. Mech. Appl. Math. 24 (1971), 155-168.

[11] F. Huang and X. Li, Convergence to the rarefaction wave for a model of radiating gas in one-dimension, Acta Math. Appl. Sin. Engl. Ser. 32 (2016), 239-256.

[12] F. Huang, A. Matsumura and X. Shi, Viscous shock wave and boundary layer solution to an inflow problem for compressible viscous gas, Comm. Math. Phys. 239 (2003), 261-285.

[13] F. Huang and X. Qin, Stability of boundary layer and rarefaction wave to an outflow problem for compressible Navier-Stokes equations under large perturbation, J. Differential Equations 246 (2009), 4077-4096.

[14] F. Huang, X. Shi and Y. Wang, Stability of viscous chock wave for compressible NavierStokes equations with free boundary, Kinetic and Related Models 3 (2010), 409-425.

[15] S. Jiang, F. Li and F. Xie, Nonrelativistic limit of the compressible Navier-Stokes-Fourier- 
P1 approximation model arising in radiation hydrodynamics, SIAM J. Math. Anal. 47 (2015), 3726-3746.

[16] S. Kawashima, Y. Nikkuni and S. Nishibata, The initial value problem for hyperbolicelliptic coupled systems and applications to radiation hydrodynamics, in: Analysis of systems of conservation laws (Aachen, 1997), Monogr. Surv. Pure Appl. Math., Vol. 99, Chapman \& Hall/CRC, (1999).

[17] S. Kawashima, Y. Tanaka, Stability of rarefaction waves for a model system of a radiating gas, Kyushu J. Math. 58 (2004), 211-250.

[18] S. Kawashima and P. Zhu, Asymptotic stability of rarefaction wave for the Navier-Stokes equations for a compressible fluid in the half space, Arch. Rat. Mech. Anal. 194 (2009), 105-132.

[19] C. Lattanzio, C. Mascia, T. Nguyen, R. Plaza and K. Zumbrun, Stability of scalar radiative shock profiles, SIAM J. Math. Anal. 41 (2009), 2165-2206.

[20] C. Lin, Asymptotic stability of rarefaction waves in radiative hydrodynamics, Commun. Math. Sci. 9 (2011), 207-223.

[21] C. Lin, J. Coulombel and T. Goudon, Shock profiles for non-equilibrium radiating gas, Phys. D 218 (2006), 83-94.

[22] C. Lin, J. Coulombel and T. Goudon, Asymptotic stability of shock profiles in radiative hydrodynamics, C. R. Math. Acad. Sci. Paris 345 (2007), 625-628.

[23] R. B. Lowrie, J. E. Morel and J. A. Hittinger, The coupling of radiation and hydrodynamics, Astrophys. J. 521 (1999), 432-450.

[24] C. Mascia, Small, medium and large shock waves for radiative Euler equations, Phys. D 245 (2013), 46-56.

[25] A. Matsumura, Inflow and outflow problems in the half space for a one-dimensional isentropic model system of compressible viscous gas, Methods Appl. Anal. 8 (2001), 645-666.

[26] A. Matsumura and M. Mei, Convergence to travelling fronts of solutions of the p-system with viscosity in the presence of a boundary, Arch. Ration. Mech. Anal. 146 (1999), 1-22.

[27] A. Matsumura and K. Nishihara, Asymptotic stability of traveling waves for scalar viscous conservation laws with non-convex nonlinearity, Comm. Math. Phys. 165 (1994), 83-96.

[28] D. Mihalas and B. W. Mihalas, Foundations of Radiation Hydrodynamics, Oxford University Press, 1984.

[29] T. Nguyen, R. Plaza and K. Zumbrun, Stability of radiative shock profiles for hyperbolicelliptic coupled systems, Phys. D 239 (2010), 428-453.

[30] X. Qin, Large-time behaviour of solutions to the outflow problem of full compressible NavierStokes equations, Nonlinearity 24 (2011), 1369-1394.

[31] X. Qin and Y. Wang, Stability of wave patterns to the inflow problem of full compressible Navier-Stokes equations, SIAM J. Math. Anal. 41 (2009), 2057-2087.

[32] X. Qin and Y. Wang, Large-time behavior of solutions to the inflow problem of full compressible Navier-Stokes equations, SIAM J. Math. Anal. 43 (2011), 341-366. 
[33] C. Rohde, W. Wang and F. Xie, Hyperbolic-hyperbolic relaxation limit for a $1 D$ compressible radiation hydrodynamics model: superposition of rarefaction and contact waves, Commun. Pure Appl. Anal. 12 (2013), 2145-2171.

[34] L. Ruan and J. Zhang, Asymptotic stability of rarefaction wave for hyperbolic-elliptic coupled system in radiating gas, Acta Math. Sci. Ser. B Engl. Ed. 27 (2007), 347-360.

[35] L. Ruan and C. Zhu, Asymptotic decay toward rarefaction wave for a hyperbolic-elliptic coupled system on half space, J. Partial Differential Equations 21 (2008), 173-192.

[36] L. Ruan and C. Zhu, Asymptotic behavior of solutions to a hyperbolic-elliptic coupled system in multi-dimensional radiating gas, J. Differential Equations 249 (2010), 26762110.

[37] D. Serre, $L^{1}$-stability of constants in a model for radiating gases, Comm. Math. Sci. 1 (2003), 197-205.

[38] X. Shi, On the stability of rarefaction wave solutions for viscous p-system with boundary effect, Acta Math. Appl. Sin. Engl. Ser. 19 (2003), 341-352.

[39] W. Vincenti and C. Kruger, Introduction to Physical Gas Dynamics, Wiley, 1965.

[40] L. Wan, T. Wang and Q. Zou, Stability of stationary solutions to the outflow problem for full compressible Navier-Stokes equations with large initial perturbation, Nonlinearity 29 (2016), 1329-1354.

[41] J. Wang and F. Xie, Singular limit to strong contact discontinuity for a 1D compressible radiation hydrodynamics model, SIAM J. Math. Anal. 43 (2011), 1189-1204.

[42] J. Wang and F. Xie, Asymptotic stability of viscous contact wave for the 1D radiation hydrodynamics system, J. Differential Equations 251 (2011), 1030-1055.

[43] F. Xie, Nonlinear stability of combination of viscous contact wave with rarefaction waves for a 1D radiation hydrodynamics model, Discrete Contin. Dyn. Syst. Ser. B 17 (2012), 1075-1100.

[44] Y. B. Zel'dovich and Y. P. Raizer, Physics of Shock Waves and High-Temperature Hydrodynamic Phenomena, Academic Press, 1967. 